\title{
eNeuro
}

Research Article: New Research / Development

\section{hop mice display synchronous hindlimb locomotion and a ventrally fused lumbar spinal cord caused by a point mutation in Ttc26}

https://doi.org/10.1523/ENEURO.0518-21.2022

Cite as: eNeuro 2022; 10.1523/ENEURO.0518-21.2022

Received: 20 December 2021

Revised: 1 February 2022

Accepted: 5 February 2022

This Early Release article has been peer-reviewed and accepted, but has not been through the composition and copyediting processes. The final version may differ slightly in style or formatting and will contain links to any extended data.

Alerts: Sign up at www.eneuro.org/alerts to receive customized email alerts when the fully formatted version of this article is published.

Copyright (@) 2022 Bernhardt et al.

This is an open-access article distributed under the terms of the Creative Commons Attribution 4.0 International license, which permits unrestricted use, distribution and reproduction in any medium provided that the original work is properly attributed. 


\section{1 hop mice display synchronous hindlimb locomotion and a}

2 ventrally fused lumbar spinal cord caused by a point mutation

3 in Ttc26

4 Short Title: Spinal CPG fusion causes synchronous gait in hop mice.

5

$6 \quad{ }^{1,2}$ Nadine Bernhardt* ${ }^{1}$ Fatima Memic*, ${ }^{1}$ Anna Velica, ${ }^{3}$ Michelle A. Tran, ${ }^{1}$ Jennifer Vieillard,

$7 \quad{ }^{5}$ Shumaila Sayyab, ${ }^{3}$ Taha Chersa, ${ }^{4,5,6}$ Leif Andersson, ${ }^{3}$ Patrick J. Whelan, ${ }^{1}$ Henrik Boije and

$8 \quad{ }^{1}$ Klas Kullander

$9 \quad *$ Contributed equally to this work, Corresponding author: klas.kullander@igp.uu.se

$11 \quad{ }^{1}$ Department of Neuroscience, Uppsala University, 75124 Uppsala, Sweden

$12{ }^{2}$ Department of Psychiatry and Psychotherapy, University Hospital Carl Gustav Carus,

13 Technische Universität Dresden, Germany.

$14{ }^{3}$ Hotchkiss Brain Institute, Department of Comparative Biology and Experimental Medicine,

15 University of Calgary, Calgary, Canada AB T2N 4N1

$16{ }^{4}$ Department of Medical Biochemistry and Microbiology, Uppsala University, Uppsala,

17 Sweden

$18{ }^{5}$ Department of Animal Breeding and Genetics, Swedish University of Agricultural Sciences,

19 Uppsala, Sweden

$20{ }^{6}$ Department of Veterinary Integrative Biosciences, Texas A\&M University, College Station,

21 Texas, USA 
23 Keywords: neuronal network, spinal cord, hop mutant, Shh signalling, patterning

24

25

26

27 Number of pages: 52

28 Words

29 Introduction: 452

30 Results: 3560

31 Discussion: 1703

32 MoM: 2604

33 Figures: 7

34 Extended data figures: 6 


\section{Abstract}

36 Identifying the spinal circuits controlling locomotion is critical for unravelling the mechanisms controlling the production of gaits. Development of the circuits governing left-

38 right coordination relies on axon guidance molecules such as ephrins and netrins. To date, no other class of proteins have been shown to play a role during this process. Here we have analyzed hop mice, which walk with a characteristic hopping gait using their hindlimbs in synchrony. Fictive locomotion experiments suggest that a local defect in the ventral spinal cord contributes to the aberrant locomotor phenotype. Hop mutant spinal cords had severe morphological defects, including the absence of the ventral midline and a poorly defined border between white and grey matter. The hop mice represent the first model where, exclusively found in the lumbar domain, the left and right components of the central pattern generators (CPGs) are fused with a synchronous hindlimb gait as a functional consequence.

These defects were associated with abnormal developmental processes, including a misplaced notochord and reduced induction of ventral progenitor domains. Whereas the underlying mutation in hop mice has been suggested to lie within the Ttc26 gene, other genes in close point mutation within Ttc26 displayed an identical morphological phenotype. Thus, our data suggest that the assembly of the lumbar CPG network is dependent on fully functional TTC26 53 protein. 


\section{Significance statement}

56 Our work reveals novel developmental defects in hop mice affecting nervous system

57 development and the assembly of local locomotor circuits. The hop mouse mutant appeared

58 spontaneously in the 1960s but the underlying cause of the unnatural synchronously hopping

59 gate has not yet been revealed. Altered functionality in hop mutant mice origins from an early

60 developmental defect in the lumbar spinal cord, resulting in a fused ventral midline. The hop

61 mouse represents an animal model harboring a very particular locomotor style that can help us

62 understand the assembly and properties of locomotor networks and how a they function to

63 coordinate motor behavior.

\section{Introduction}

65 In the early 20th century, Thomas Graham-Brown demonstrated that the spinal cord could

66 generate the basic pattern for stepping without descending or peripheral input (Graham-

67 Brown T, 1911). Within his model of spinal locomotor control, he implemented Sherrington's

68 term "half-center" for two groups of reciprocally organized neurons mutually inhibiting each

69 other to provide a coordinated pattern for stepping. Such local spinal networks, capable of

70 generating rhythmic motor output, are referred to as central pattern generators (CPGs).

71 Studies on the spinal cord locomotor CPG have identified key principles for the development

72 and function of neuronal circuitry. For example, mouse mutants with abnormal locomotor

73 coordination have been informative for understanding CPG organization and function

74 (Kullander et al., 2001, 2003; Lanuza et al., 2004; Akay et al., 2006; Lundfald et al., 2007;

75 Crone et al., 2008, 2009; Zhang et al., 2008; Rabe et al., 2009; Zagoraiou et al., 2009; 
Restrepo et al., 2009; Andersson et al., 2012; Talpalar et al., 2013; Lemieux et al., 2016; Thiry et al., 2018).

The CPGs controlling hindlimb muscle activity during locomotion are located in the ventral part of the lumbar spinal cord (Bracci et al., 1996; Kjaerulff and Kiehn, 1996) whereas CPGs controlling forelimb activity are located in the cervical spinal cord. Inhibitory actions between CPGs on different rostrocaudal levels of the spinal cord coordinate ipsilateral flexor and extensor muscles. Each side of the midline contains CPGs capable of rhythm generation where commissural inhibitory and excitatory projections ensure left-right coordination (Bannatyne et al., 2003; Butt and Kiehn, 2003; Hammar et al., 2004; Jankowska, 2008; Lanuza et al., 2004; Quinlan and Kiehn, 2007; Talpalar et al., 2013). Left-right activity persists when the dorsal spinal cord is removed, whereas left-right alternating fictive locomotion degrades after cutting the spinal cord ventral commissure. These experiments illustrate that ventromedial commissural interneurons (CINs) are critical for bilateral coordination (Andersson et al., 2012; Kjaerulff and Kiehn, 1996; Restrepo et al., 2009). Neuronal connections form during embryonic development when differentiating neurons send their axons, navigating through the embryonic environment to synaptic targets. This process is mediated by conserved families of axon guidance proteins including netrins, ephrins, slits and semaphorins (Vallstedt and Kullander, 2013) and impaired axon guidance in the spinal cord results in locomotor coordination dysfunction (Kullander et al., 2001, 2003; Rabe et al., 2009; Rabe Bernhardt et al., 2012; Satoh et al., 2016; Wegmeyer et al., 2007). In the present study, we analyzed hop mice that walk with a characteristic hopping gait using their hindlimbs in synchrony. Mice carrying the hop-sterile (hop or hydrocephalicpolydactyly, hpy) mutation appeared spontaneously in 1967 in the C57BL/10J strain, and the mutation was first found to be localized at the proximal end of chromosome 6 (Bryan et al., 1977; Hollander, 1976; Johnson and Hunt, 1971; Lyon, 1973). Later a point mutation within 5 

the Ttc26 gene was identified and suggested to be responsible for impaired hedgehog

102 signaling (Swiderski et al., 2014). However, since other genes in the close vicinity have been

103 associated with gait defects, it remained unclear whether the point mutation is causative for

104 the hop locomotor phenotype. Using CRISPR technology we introduced a single point

105 mutation within the Ttc26 gene, which reproduced the anatomical phenotype observed in hop

106 mice. Further, we show that the synchronous gait in hop mice is the result of a left and right

107 CPG component fusion exclusively within the lumbar domain of the spinal cord. This is in

108 turn caused by abnormal notochordal and ventral spinal cord signaling during development,

109 resulting in migration defects and an absent ventral midline.

110 


\section{Material and Methods}

\section{Animals}

113 Mice

114 CByJ.Cg-hop/J mice were imported from Jackson Laboratory (N12 on a backcross-intercross

115 to BALB/cByJ). The colony was kept on the BALB/cByJ background for analysis of the

116 locomotor phenotype and outcrossed 2-4 generations to the C57BL/6J background for

117 positional cloning. Ttc26 $6^{\mathrm{Y430X}}$ mutant mice were produced in the C57BL/6J background by

118 Siu-Pok Yee at the Gene Targeting and Transgenic Facility, Uconn Health Center. All

119 experiments involving animals were approved by the appropriate local Swedish ethical committee (C147/7 or C79/9), and by the University of Calgary Health Sciences Animal Care

121 Committee.

\section{Gait study}

124 2-3 month old animals were trained to walk on a 1 meter long and $10 \mathrm{~cm}$ wide track the day

125 before the experiment. Mice were taken up and painting of the paws was mimicked with a

126 brush. Each mouse had to go to the end of the track at least 3 times without stopping. The day

127 of the experiment mice were handled exactly the same as during the training but this time the

128 paws were painted with two distinct colors for the forelimbs (red) and hindlimbs (blue).

129 Footprints were recorded on Whatmann paper placed at the bottom of the track. For each

130 animal, 3 runs were recorded and analyzed according to (Kullander et al., 2001). 


\section{Electrophysiology}

133 Experiments were performed on 0-3 days old (P0-P3, weight $1.16 \mathrm{~g}$ to $4.05 \mathrm{~g} ; \mathrm{n}=62)$ hop

134 homozygote, heterozygote and wild type control mice. Mutant mice were identified by

135 preaxial polydactyly of the hindfeet (Fig. 1-1) and the synchronous activity in their hindlimbs

136 when suspended in the air with their tails lightly pinched. There were no obvious deficits in gait in the hop heterozygote and wild type control mice.

For in vivo experiments, animals were anaesthetized by hypothermia and then suspended in a sling such that the fore- and hindlimbs moved freely (Norreel et al., 2003). Electromyographic (EMG) electrodes were inserted into the left and right tibialis anterior muscles parallel to the muscle fibres, and a grounding electrode was inserted subcutaneously into the back. EMG electrodes were made of 75 mm Teflon-coated platinum-iridium wires (A-M Systems Inc.). A heat lamp was used to keep the air temperature at $30^{\circ} \mathrm{C}$. Recordings were amplified (1000 times), bandpass filtered (100 - $1 \mathrm{kHz}$ ), and digitized at $3 \mathrm{kHz}$ (Axon Digidata 1322A) for future analysis. 5 - 10 minutes after the EMG wires were inserted, air-stepping sequences were elicited by pinching the tail with forceps and the activity was recorded. For in vitro experiments, the animals were anaesthetized by hypothermia. Animals were rapidly decapitated, eviscerated and the remaining tissue was placed in a dissection chamber filled with oxygenated $\left(95 \% \mathrm{O}_{2}-5 \% \mathrm{CO}_{2}\right)$ artificial cerebrospinal fluid (ACSF:

concentrations in mM: $128 \mathrm{NaCl}, 4 \mathrm{KCl}, 0.1 \mathrm{CaCl}_{2}, 2 \mathrm{MgSO}_{4}, 0.5 \mathrm{Na}_{2} \mathrm{HPO}_{4}, 21 \mathrm{NaHCO}_{3}, 30$ D-glucose). A ventral laminectomy exposed the cord, and the ventral and dorsal roots were cut. The spinal cord was transected at thoracic $1-3\left(\mathrm{~T}_{1-3}\right)$ to sacral $2-3\left(\mathrm{~S}_{2-3}\right)$ and carefully removed from the vertebral column. After 30 minutes, the preparation was transferred to the recording chamber and superfused with oxygenated ACSF (concentrations in mM: $128 \mathrm{NaCl}$, $4 \mathrm{KCl}, 1.5 \mathrm{CaCl}_{2}, 1 \mathrm{MgSO}_{4}, 0.5 \mathrm{Na}_{2} \mathrm{HPO}_{4}, 21 \mathrm{NaHCO}_{3}, 30$ D-glucose). The bath solution was 8 
then heated gradually from room temperature to $27^{\circ} \mathrm{C}$. The preparation was allowed to acclimate for one hour thereafter. Population motoneuron bursting activity was recorded using suction electrodes into which segmental ventral roots from the left and right lumbar $L_{2}$ and $L_{5}$ segments were drawn (Whelan et al., 2000). In some experiments we found that the $\mathrm{L}_{2}$ ventral root was not well formed and in these cases we recorded from the $\mathrm{L}_{3}$ ventral root. Neonatal mice show synchrony between $\mathrm{L}_{1}, \mathrm{~L}_{2}$, and $\mathrm{L}_{3}$ ventral root neurograms (Whelan et al 2000). The resultant neurograms were amplified (100 - 20,000 times), band pass filtered (100 Hz - 1 $\mathrm{kHz}$ ) and digitized at $2-5 \mathrm{kHz}$ (Axon Digidata 1320) for future analysis. Ten minutes of subtracted from the processed data and further smoothed using a digital filter (Savitzky-

control baseline activity was recorded prior to adding drugs. $5 \mu \mathrm{M} \mathrm{N}$-methyl-DL-aspartic acid (NMA, Sigma-Aldrich), $10-20 \mu \mathrm{M}$ Serotonin (5-HT, Sigma-Aldrich) and $50-75 \mu \mathrm{M}$ dopamine (DA, Sigma-Aldrich) were added to the bath and the rhythm stabilized for 10 - 30 minutes. In some experiments, a GABA uptake inhibitor (0.1 - 1 mM nipecotic acid or 50 $100 \mu \mathrm{M}$ NO-711, Sigma-Aldrich) and/or a glycine uptake inhibitor (100 $\mu \mathrm{M}$ sarcosine, Sigma-Aldrich) was added to the bath solution. In some experiments, we examined whether a hemisected spinal cord was capable of generating coordinated rhythmic activity. In these experiments, we first examined the activity patterns in the presence of rhythmogenic drugs. We then removed the recording electrodes and using a pair of micro-clippers completely midsagittally hemisected the spinal cord. The suction electrodes were then reattached to the ventral roots.

Data were digitally rectified, integrated and then analyzed using custom written programs (MatLab, MathWorks, Natick, MA). Locomotor-like activity was quantified using time series analysis. Time series analysis was performed by taking intervals of 60 seconds of raw data, rectifying the data, applying a low-pass filter and resampling at $100 \mathrm{~Hz}$. Means were Golay, $3^{\text {rd }}$ order polynomial operating over 13 points). Cross and auto-correlograms were then 9 
calculated and the quality of the rhythm was assessed by measuring the correlation coefficients for the segmental $L_{2}$ ventral root bursts and the left $L_{2}$ and left $L_{5}$ ventral root bursts. To measure rhythm stability, the peak-to-trough correlation coefficient (PTCC) was calculated from the cross correlogram by subtracting the minimum negative value of the correlation coefficient from the maximum of the first positive peak over the first 75 lags (each lag $=50 \mathrm{~ms}$ ). Stable synchronous rhythms typically have high positive correlation coefficients at zero phase lag. The cycle periods for the resultant rhythm were calculated by measuring the number of lags from one peak to the next from the auto-correlogram. The phase lag between ventral root bursts was obtained from the cross correlogram and was defined as the distance from the minimum trough around lag 0 to the next peak divided by the cycle period (Madriaga et al., 2004). Data are expressed as mean \pm standard error of the mean and significance was analyzed using paired and unpaired Student's t-tests if the data were normally distributed $(\mathrm{P}<0.05)$. Data that were not normally distributed were analyzed using a Wilcoxon-Signed Rank test. Multiple data points were analyzed using a one-way ANOVA followed by a Tukey post hoc test to detect significant differences. To analyze in vivo EMG data and to illustrate phase relationships for selected in vitro experiments, we used circular statistics, in which the phase was normalized from 0 to 1 (Drew and Doucet, 1991). If the length of the arrow is large this suggests a tendency for the rhythms represented by the two neurograms to be coupled. Significance was computed using Rayleigh's test $(\mathrm{P}<0.05)$.

\section{Tracing of commissural interneurons}

Fluorescent dextran-amines 3,000 MW rhodamine-dextran-amine (RDA) and 3,000 MW fluorescein-dextran-amine (FDA) (Invitrogen, Sweden) were used for retrograde tracing of commissural interneurons (CINs) as described previously (Eide and Glover, 1995). P0-P3 mice spinal cords were prepared as described earlier (Wegmeyer et al., 2007). Tracings on 10 
E12.5 embryos were performed essentially the same way, but the spinal cord remained within the vertebral column during tracer application and incubation. Preparations were incubated for 12-16 $\mathrm{h}$ and then fixed in 4\% paraformaldehyde (PFA) in 0.1 M phosphate-buffered saline (PBS), pH 7.4 and stored dark at $4{ }^{\circ} \mathrm{C}$ for 1 week. Spinal cords were cut into $60 \mathrm{~mm}$ thick transverse sections on a vibratome (Leica, Germany) and stored in the dark at $-20^{\circ} \mathrm{C}$ until analysis. Embryonic tracings were fixed for 2 hours, transferred to $30 \%$ sucrose in PBS at $4^{\circ} \mathrm{C}$ over-night, embedded in OCT and $12 \mathrm{~mm}$ sections were cut using a cryostat (CM1800, Leica), collected onto Superfrost slides (Menzel-Gläser, Germany)

\section{In situ hybridization}

Free floating vibratome sections were rehydrated in consecutive washes for $10 \mathrm{~min}$ in $75 \%$, 50\% and 25\% methanol in PBT, bleached in 6\% hydrogen peroxide in PBT for 15 min and treated with $0.5 \%$ Triton X-100 for 5 min. The sections were digested with proteinase K (10 $\mu \mathrm{g} / \mathrm{ml}$ ) in PBT for 15 min. The digestion was stopped with a wash in glycine (Scharlau Chimie, Spain; 2mg/ml) in PBT for 5 min, and sections were postfixed in $4 \%$ formaldehyde for $20 \mathrm{~min}$. The sections were prehybridized at $65^{\circ} \mathrm{C}$ in hybridization buffer ( $50 \%$ formamide, 5xSSC pH 4.5, 1\% SDS, $50 \mu \mathrm{g} / \mathrm{ml}$ tRNA (Sigma) and $50 \mu \mathrm{g} / \mathrm{ml}$ heparin (Sigma)) for two hours prior to addition of probe. $1 \mu \mathrm{g} / \mathrm{ml}$ probe was added to the hybridization buffer and sections were hybridized over-night at $65^{\circ} \mathrm{C}$. Excess probe was removed by washes in wash buffers (50\% formamide, 5xSSC pH 4.5 and 1\% SDS; 50\% formamide, 2xSSC pH 4.5 and $0.1 \%$ tween-20) at $65^{\circ} \mathrm{C}$ for 3 times 30 min each. The sections were transferred to blocking solution (1\% blocking reagent in TBST) for $2 \mathrm{~h}$ before addition of anti-DIG AP (1:5000) diluted in blocking solution and incubated over-night at $4^{\circ} \mathrm{C}$. The sections were treated with levamisole $(0.5 \mathrm{mg} / \mathrm{ml})$ in TBST and levamisole $(0.5 \mathrm{mg} / \mathrm{ml})$ in NTMT (100 mM NaCl, 10 
$\mathrm{mM}$ Tris- $\mathrm{HCl} \mathrm{pH}$ 9.5, $50 \mathrm{mM} \mathrm{MgCl}_{2}$ and $0.1 \%$ Tween-20) before developing in BM purple AP substrate (Roche) at $37^{\circ} \mathrm{C} 1 \mathrm{~h}-4$ days. Between additions of new chemicals, sections were washed with either PBT (prior to addition of probe) or TBST (after addition of probe). In situ hybridization on cryosections was performed essentially as described (Schaeren-Wiemers and Gerfin-Moser, 1993). The VIAAT probe covers nucleotides 588-2072, the Vglut2 probe 1616 -2203 used as described earlier (Wallén-Mackenzie et al., 2006), the VAChT probe 1534 2413, Netrin-1 and EphrinB3 probe 1-1021.

\section{Immunohistochemistry}

The tissue was cryoprotected in 30\% sucrose in PBS, embedded in OCT medium and sectioned at a thickness of 12-14 $\mu \mathrm{m}$ on a cryostat (CM1800, Leica). The sections were washed in PBS and pre-blocked in blocking solution - 5\% goat serum, 0.3\% Bovine Serum Albumin (BSA, Sigma Aldrich) and 0.1\% Triton ${ }^{\circledR}$ X-100 (Sigma Aldrich) in PBS. Primary antibodies were diluted in blocking solution, added to the sections and incubated over night at $4^{\circ} \mathrm{C}$. The following dilutions were used: mNkx2.2 1:100 (Hybridoma bank), mEvx1 1:50 (Hybridoma bank), gpLbx1 1:10000 (kind gift from C. Birchmeyer), rPax2 1:1000 (Covance), mBrn3a 1:500 (Chemicon), mIsl1/2 1:100 (Hybridoma bank), mLhx1/5 1:500 (Hybridoma bank), Lhx2/9 1:8000 (kind gift from Dr. T.Jessell), mShh 1:100 (Hybridoma bank), mMap2 1:500 (Chemicon, Sweden), rbNeuN 1:1000 (Chemicon), MBP 1:500 (AbCam), rbTtc26 1:1000 (Novus Biologicals), mNeuN 1:500 (Chemicon), gpVAChT 1:500 (Millipore), gChAT 1:250 (Millipore), mParvalbumin 1:1000 (Sigma) and gpDmrt3 1:10 000 (custom made using the immunizing peptide CKQSIYTEDDYDERS-amide).

For some antibodies, antigen retrieval was necessary in order to get good immunohistochemical staining. The sections were then washed with PBS and thereafter placed in a metal rack inside a beaker filled with preheated citrate buffer ( $9 \mathrm{ml} 0.1 \mathrm{M}$ citric 12 


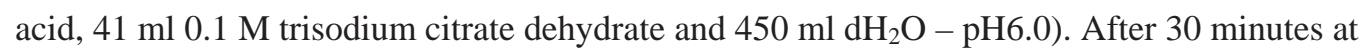

$25698^{\circ} \mathrm{C}$, the beaker was left to cool down at room temperature for 30 minutes. Subsequently the

257 sections were washed with $\mathrm{dH} 2 \mathrm{O}$ for 1 minute and pre-blocked in $1 \%$ normal goat serum and

$2580.1 \%$ Triton ${ }^{\circledR} \mathrm{X}-100$ in PBS for 1 hour. Finally, the primary antibodies were diluted in

259 blocking solution (1\% normal goat serum and $0.1 \%$ Triton ${ }^{\circledR} \mathrm{X}-100$ in PBS), added to the

260 sections and incubated over night at $4^{\circ} \mathrm{C}$. The following day, the sections were washed with

261 PBS and incubated with DAPI (Sigma Aldrich, 200ng/ml) and the secondary antibodies

262 (diluted 1:500 in blocking solution) for 2-3 hours at room temperature. After washing with

263 PBS, the sections were mounted in Mowiol $^{\circledR}$ 4-88 (Sigma Aldrich). Secondary antibodies

264 used: goat anti-mouse conjugated with FITC 488 (F(ab’) 2 Fragment, 111-096-003, Jackson

265 Immunoresearch Labs), donkey anti-guinea pig conjugated with Alexa 594 (Jackson

266 Immunoresearch Labs), donkey anti-rabbit conjugated with Alexa 488 (Invitrogen) and

267 donkey anti-goat conjugated with Alexa 488 (Jackson Immunoresearch Labs).

$268 T$ Ttc26 ${ }^{\text {Y430X }}$ mice embryos (E16.5) were euthanized, genotyped, fixed in 4\% paraformaldehyde

269 for 4 hours on a rocking table at room temperature and then washed in $0.1 \mathrm{M}$ phosphate

270 buffered saline (PBS - Sigma Aldrich, Stockholm, Sweden) for 30 minutes before being

271 cryoprotected in 30\% sucrose in PBS. The tissue was shipped in 30\% sucrose in PBS (in a

272 falcon tube placed inside a Styrofoam box with cool-packs) to Uppsala University. Upon

273 arrival 5 days later the embryos were frozen in OCT medium (Richard Allan Scientific

$274 \mathrm{NEG50}^{\mathrm{TM}}$ ) on dry ice. Cryostat sections were cut (cryostat CM1800, Leica) at a thickness of

$27514 \mu \mathrm{m}$ and placed on Superfrost slides (Menzel-Gläser, Germany). For the

276 immunohistochemistry experiment, postnatal P2 wild type rabbits from the Sauteur d'Alfort

277 breed were obtained from the Fédération Française de Cuniculiculture. They were

278 anesthetized with a mix of medetomidine (Dormilan ${ }^{\circledR} 0,1 \mathrm{ml} / \mathrm{kg}$ ) and ketamine (Ketamidor ${ }^{\circledR}$

$2790,15 \mathrm{ml} / \mathrm{kg}$ ) and cardiac perfusion was performed with PBS followed by formaldehyde $4 \%$ in 13 
280

281

282

283

284

285

286

287

288

289

290

291

292

293

294

295

296

297

298

299

300

301

302

303

304

PBS. The spinal cords were dissected and postfixed overnight at $4{ }^{\circ} \mathrm{C}$ in formaldehyde $4 \%$ in PBS.

\section{Histology staining}

For haematoxylin and Eosin staining, cryosections of spinal cord were rehydrated in $\mathrm{dH}_{2} \mathrm{O}$ for 1 minute, stained with progressive haematoxylin (Mayer's) for 90 seconds, washed twice in $\mathrm{dH}_{2} \mathrm{O}$ for 1 minute, dipped in Scott's tap water $\left(20 \mathrm{~g} \mathrm{MgSO}_{4} \cdot 7 \mathrm{H} 2 \mathrm{O}\right.$ and $2 \mathrm{~g} \mathrm{NaHCO}$ in $1 \mathrm{~L}$ tap water), dehydrated in 96\% Ethanol in $\mathrm{dH} 2 \mathrm{O}$ for 1 minute and finally counter-stained with 0.5\% Eosin (Sigma Aldrich) in $\mathrm{dH}_{2} \mathrm{O}$ for 20 seconds. Afterwards the sections were dehydrated with successive washes in 70\% and $96 \%$ Ethanol in $\mathrm{dH} 2 \mathrm{O}$ for 1 minute and treated with X-tra Solve ${ }^{\circledR}$ (MEDITE) for 4 minutes before being mounted in Pertex® (Histolab®). 3,3'-diaminobenzidinetetrahydrochloride (DAB) (Sigma, St Louis, MO, USA) was used on free-floating spinal cord sections to visualize white matter (dark brown) and Luxol fast blue (LFB) was used to analyze paraffin embedded brain sections staining myelin and phospholipids (blue-green) counterstained with cresyl violet as previously described (Kullander et al., 2001).

For the histology experiment, wild adult rabbits were euthanized in Stockholm by Stockholms Vilt- och Skadedjursgrupp and rabbit tissue was shipped on ice to Uppsala University within a few hours. Upon arrival, the vertebral column was placed in 4\% paraformaldehyde in PBS for two weeks, before the spinal cord was dissected. Thereafter the tissue was cryoprotected in 30\% sucrose in PBS for 60 hours and frozen in OCT medium (Richard Allan Scientific NEG50TM) on dry ice. 


\section{CRISPR mutagenesis}

307 Based on previously published data (32) and our independent exome sequencing validation

308 (Fig. 7-1), a point mutation located at Chr6:38,362,071 (C>A) and resulting in a nonsense 309 mutation (Y430X) within the Tetratricopeptide repeat protein 26 (Ttc26) gene was identified 310 in hop mice. We used CRISPR/Cas9-mediated genome editing technology with the help from 311 Siu-Pok Yee, Ph.D. at the Gene Targeting and Transgenic Facility, Uconn Health Center. A 312 C/A transversion at position 38412065 in exon 15 of the gene Ttc26 on chromosome 6 was to create a novel PvuII site, 3' of the knock-in termination codon in Ttc26 to allow for straightforward genotyping of the mice by PCR followed by a PvuII digestion. The PvuII site also affected the PAM sequence of the CRISPR so re-digestion of the KI allele was avoided. CAS nuclease, a guide RNA and an oligonucleotide carrying the desired mutation and homology arms were co-injected into the nuclei of fertilized oocytes, followed by implantation of the eggs into surrogate mothers to obtain offspring. An allele-specific primer was developed for genotyping of the pups. We obtained one Ttc26 Y430X founder female that appeared to be heterozygous for the mutation (1:1 peak ratio from sequencing, Fig 5A). From F1 pups from the founder female, we bred heterozygous males and females to obtain 324 homozygous Ttc26 $6^{\text {Y430X }}$ mouse mutants.

\section{Imaging and picture processing}

327 Fluorescent and bright field images were acquired either on an Olympus BX61WI microscope 328 (Olympus, Sweden) using the Volocity software (Improvision, Lexington, USA) or on a 329 MZ16F dissection microscope with a DFC300FX camera and FireCam software (Leica). 15 
Close-ups were taken using the OptiGrid Grid Scan Confocal Unit (Qioptiq, Rochester, USA) or a confocal microscope (Zeiss LSM 510 Meta, Oberkochen, Germany). For illustrations, captured images were auto-leveled using Adobe Photoshop software. Quantitative image analysis was performed using the Fiji processing suite (REF). For each image, region of interests were manually defined. For quantification of fluorescence, a global threshold was applied before relevant object information was extracted. Distribution plots were obtained using R Studio Version 4.0.4 (R Core Team (2021).) and the spatstat package (Baddeley and Turner, 2005).

\section{Multiple sequence alignments of TTC26}

An amino acid sequence analysis of TTC26 was performed to generate multiple sequence alignment. In order to perform this analysis, we first downloaded the protein sequences from the National Center for Biotechnology Information (NCBI) for multiple species (Human, Rabbit, Dog, Cow, Pig, Chimpanzee, Mouse and Rat) with the accession number (NP_079202.2, XP_002722121.2, XP_003639595.2, XP_002687045.1, XP_003134668.2, XP_527905.3, NP_705828.2, NP_001020216.1) respectively. Multiple sequence alignments was performed using MUSCLE software (Edgar 2004, PMID: 15034147) with default parameters, in the multiple sequence alignment editor SEAView (Galtier 1996, PMID:

9021275) version 3.2 by setting the human sequence as the reference with the block size of 10.

\section{Statistical analysis}

Simple effects of genotype (crtl, hop) were analyzed using independent t-test (two-tailed) or Mann-Whitney U test dependent on data distribution. Analyses were performed using MS Excel 2016 or SPSS Statistics (version 27.0, IBM, Armonk, NY, USA). Data was plotted 16 
using R (https://www.r-project.org) and the package shiny and vioplot. Statistical significance was set at $\mathrm{p}<.05$, effect sizes are reported with Cohen's $\mathrm{d}$ or $\mathrm{r}$ (rank-biserial correlation) for non-parametric testing.

\section{Results}

\section{Aberrant synchronous hindlimb coordination in hop mice correlates to local spinal cord} neuronal circuitry

Homozygous hop mutants show distinct phenotypes such as preaxial polydactyly of all feet, sperm tail deficiency resulting in male sterility, and hydrocephalus (Hollander, 1976; Johnson and Hunt, 1971; Swiderski et al., 2014). Although there have been no detailed reports on the locomotor pattern produced by hop mutant animals, it has been described that they exhibit a characteristic hopping gait (Johnson and Hunt, 1971; Wojnowski et al., 1998). We confirmed and extended these observations by conducting gait analysis and electrophysiological experiments. Gait analysis in adults revealed that during locomotion, mice homozygous for the hop mutation, herein referred to as hop mice, alternated their forelimbs while their hindlimbs moved in synchrony (Fig. 1A, B). To assess the onset of this hopping pattern, we evoked locomotor activity by pinching the tail, followed by electromyographic (EMG) recordings from the tibialis anterior muscles in conscious early postnatal (P0-P5) hop and control animals. We found that hop mice produced an air locomotor pattern of synchronous hindlimb movements. As expected, control neonatal mice produced a typical left-right alternating pattern (Fig. 1C-E).

To determine whether deficiencies in the spinal cord CPG are the sole cause of the locomotor phenotype, we performed experiments using isolated spinal cord preparations. Following bath application of 5-HT, DA and NMDA, we found that in the majority of hop mutants (20/28), a 
synchronous fictive locomotor rhythm developed between left-right ventral roots. In contrast, the alternating ipsilateral flexion-extension like pattern between the L2 and L5 roots was preserved (Fig. 1F-H). We observed that in contrast to control mice the L5 burst duration was extended and an asymmetry was observed between the L2 and L5 burst duration in 13/20 hop mutants. The data represents an average for 5 min following the development of a sustained rhythm (L2- L2: $1.10 \pm 0.06$ (PTCC), $5.61 \pm 0.68$ s (cycle period), $0.05 \pm 0.01$ (phase lag); L2-L5: $-1.04 \pm 0.06$ (PTCC), $0.52 \pm 0.03$ (phase lag), $n=20$ ). Although the dominant rhythm observed was a synchronous left-right and an ipsilateral alternating pattern, it was not the only one. 5/28 hop mutant mice exhibited a left-right alternating pattern consistent with a normal locomotor-like pattern. In 3/28 hop mutant mice, we observed a synchronous pattern between all roots ( $\mathrm{L}_{2}-\mathrm{L}_{2}: 1.26 \pm 0.10$ (PTCC), $11.78 \pm 3.76 \mathrm{~s}$ (cycle period), $0.01 \pm 0.01$ (phase lag); $\mathrm{L}_{2}-\mathrm{L}_{5}: 1.14 \pm 0.13$ (PTCC), $0.01 \pm 0.003$ (phase lag)). Moreover, the data indicate that the fictive locomotion in hop mice was less stable than in control mice, and in 5 of the 20 animals that displayed a synchronous segmental left-right and ipsilateral alternating rhythm, it was sustained for around 5 minutes, which was notably different from control mice, where the standard alternating rhythm was sustained for up to $60 \mathrm{~min}$. There were no significant differences in cycle periods between hop mutants and controls $\left(\mathrm{L}_{2}: 5.19 \pm 0.39 \mathrm{~s}, \mathrm{~L}_{5}: 5.53 \pm\right.$ 0.37 s (mutants); $\mathrm{L}_{2}: 5.83 \pm 0.98 \mathrm{~s}, \mathrm{~L}_{5}: 5.33 \pm 0.80 \mathrm{~s}$ (controls); $\mathrm{n}=8$; $\mathrm{P}>0.5$ ). The synchronous fictive locomotion we observed in 20/28 hop mice was in accordance with the in vivo gait analyses as well as the EMG recordings.

In the 20 animals that generated synchronous segmental patterns and ipsilateral alternation, we found that the ipsilateral pattern of $\mathrm{L}_{2 / 3}-\mathrm{L}_{5}$ alternation was maintained following hemisection, suggesting that the circuitry coordinating flexor-extensor alternation was intact in these hop mutants (Fig. 1-1). Following hemisection, it was usually necessary to increase the concentration of the drug cocktail (DA to $75 \mu \mathrm{M}$, 5-HT to $15 \mu \mathrm{M}$ ) to maintain rhythmic activity [ $\mathrm{L}_{2 / 3}-\mathrm{L}_{5}: 1.18 \pm 0.15$ (PTCC), $5.53 \pm 3.03 \mathrm{~s}$ (cycle period), $0.51 \pm 0.04$ (phase lag), $\mathrm{n}=2$ ]. The increase in concentration is typical of previous reports (Whelan et al., 2000). 
The asymmetry between flexor and extensor bursts was not present in the hemisected cords (Fig. 1-1B), and was qualitatively similar to reports using normal mice (Whelan et al., 2000).

To further establish whether synchronous left-right rhythmic activity was the dominant pattern, we used electrical stimulation of the cauda equina or dorsal roots to elicit a rhythm (Gordon and Whelan, 2006; Whelan et al., 2000). We found that in hop mice, electrical stimulation did evoke a synchronous rhythm similar to that observed using pharmacological stimulation (Fig. 1-1C). In addition, in several mice, we were able to observe spontaneous bouts of synchronous left-right and alternating $\mathrm{L}_{2}-\mathrm{L}_{5}$ rhythm that would last a few seconds. Collectively, these data support the idea that the hop deficit is at least partly due to alterations in the lumbar network.

Previous studies have shown that neurotransmitter balance over the midline is critical for coordinated left-right output of the CPG, and during fictive locomotion, the balance between excitation and inhibition can be shifted using pharmacological strengthening (Hinckley et al., 2005; Juvin et al., 2005; Kullander et al., 2003; Wu et al., 2018). Following administration of nipecotic acid or NO-711 (data pooled since no difference was observed between the two drugs), we found that, although the left-right pattern did not switch to an alternating pattern, the rhythm became less stable (Fig. 1I, middle panel; [control: $\mathrm{L}_{2}-\mathrm{L}_{2}: 1.11$ \pm 0.06 (PTCC), $6.34 \pm 1.56$ s (cycle period), $0.03 \pm 0.01$ (phase lag); $L_{2}-L_{5}:-1.10 \pm 0.04$ (PTCC), $0.49 \pm 0.04$ (phase lag); with nipecotic acid or NO-711 (pooled data): $\mathrm{L}_{2}-\mathrm{L}_{2}: 0.75 \pm$ 0.33 (PTCC), $4.88 \pm 0.55$ s (cycle period), $0.11 \pm 0.06$ (phase lag); $\mathrm{L}_{2}-\mathrm{L}_{5}:-1.01 \pm 0.10$ (PTCC), $0.73 \pm 0.13$ (phase lag); $n=7]$. We also tested both sarcosine and nipecotic acid bath applied simultaneously in a separate series of experiments. We found that this manipulation consistently altered network dynamics by changing the synchronous pattern to a less stable pattern, which at times appeared to be alternating (Fig. 1I right panel; [control: $\mathrm{L}_{2}-\mathrm{L}_{2}: 1.29 \pm$ 0.13 (PTCC), $3.73 \pm 0.35$ s (cycle period), $0.06 \pm 0.003$ (phase lag); $\mathrm{L}_{2}-\mathrm{L}_{5}:-1.17 \pm 0.07$ (PTCC), $0.58 \pm 0.03$ (phase lag); with sarcosine and nipecotic acid: $\mathrm{L}_{2}-\mathrm{L}_{2}: 0.27 \pm 0.44$ (PTCC), $4.54 \pm 0.23$ s (cycle period), $0.24 \pm 0.15$ (phase lag); $\mathrm{L}_{2}-\mathrm{L}_{5}:-1.05 \pm 0.10$ (PTCC), $0.55 \pm 0.04$ (phase lag); $n=3]$ ). 

pattern previously reported and that defects in the lumbar spinal cord circuitry are sufficient to explain the altered hindlimb coordination. Further, whereas changing the balance of excitatory and inhibitory connections over the midline in hop mice caused altered network activity, the synchronous left-right output was not consistently switched back to alternation.

\section{Altered spinal cord morphology in hop mice}

Our electrophysiological experiments suggested that the aberrant locomotor phenotype arose from local spinal neuronal circuitry output, therefore we examined the morphology of the spinal cord. The gross morphology of wild type postnatal spinal cords is characterized by a cervical and lumbar enlargement, which contain, among others, the neurons that coordinate fore- and hindlimb movement, respectively. The cervical enlargement extends from C5 to T1, and the lumbar enlargement extends from L2 to L6. The spinal cord from early postnatal control mice was found to measure $14 \pm 3 \mathrm{~mm}$. In hop mice, the length of the spinal cord, 14 $\pm 5 \mathrm{~mm}$, and the size of the cervical enlargement were comparable to controls, whereas the lumbar enlargement was hardly detectable (Fig. 2A-B). Further, wild type spinal cords had a visible ventral spinal artery fed by a radicular artery termed the artery of Adamkiewicz, which in wild type mice usually originates at the level of $\mathrm{L}_{1-2}$ (Fig. 2A, arrow). In the lumbar region of hop spinal cords, the blood supply was abnormal, including the absence of a ventral spinal artery together with an undefined artery of Adamkiewicz (Fig. 2A, star).

$$
\text { Next, we used the spinal cord open-book preparation, in which the spinal cord is cut }
$$
dorsally along the rostrocaudal axis, and the left and right halves of the spinal cord are dissected to reveal the inside of the two halves and the remaining ventral part. In this preparation, using NeuN as a marker for neurons, we found that the morphology of the 
positive neurons and the ventral area, whereas the more rostral and caudal parts were unaffected (Fig. 2C,D). Transverse sections of lumbar spinal cords stained with NeuN (grey matter) and myelin basic protein (MBP) as a marker for myelinated axons (white matter), revealed less myelinated structures and misplaced neurons in hop mutants (Fig. 2E,F).

Distribution plots obtained from these stainings further illustrate the misplacement of neurons along the medio-lateral axis (magenta) and a dorsally shifted central canal (blue) in hop mice (Fig. 2G,H).

Similarly, adult mice transverse sections of the cervical spinal cord from hop mice showed no morphological differences compared to controls (Fig. 2I). However, sections of lumbar spinal cords from hop mice showed an altered morphology in the ventral spinal cord, including an undefined border between white and grey matter, the absence of a ventral funiculus and a dorsally shifted, poorly delineated, and smaller central canal (Fig. 2I-L). Immunohistochemistry, using NeuN and MBP antibodies, demonstrated misplaced neurons within the white matter also in adult hop mutants (Fig. 2M-O). Other mouse mutants with locomotor phenotypes have been reported to display severe defects in several brain regions, including the corpus callosum, hippocampal-, anterior-, habenular- and posterior commissure as well as the pontine nucleus and cerebellar structures (Ackerman et al., 1997; Chiang et al., 1996; Fazeli et al., 1997; Kullander et al., 2001; Páez et al., 2007; Serafini et al., 1996). In contrast, morphological analysis of brains from adult hop mice, which display enlarged ventricles likely connected to the hydrocephalic phenotype, did not show any further brain abnormalities (Fig. 2-1).

Together, these findings suggest that the synchronous gait in hop mice is the result of ventral spinal cord alterations limited to the lumbar region responsible for controlling the hindlimbs. The rostro-caudal specificity is further supported by the observed forelimb alternating locomotion and normal cervical spinal cord morphology. 
485 Aberrant commissural fibres and neurotransmitter phenotype in hop mice

486 Cross-inhibitory and excitatory actions from CINs between the two sides of the locomotor

487 CPG ensure coordinated left-right movements (Cowley and Schmidt, 1995; Kjaerulff and

488 Kiehn, 1996). In an effort to further explain the aberrant locomotor phenotype, we examined

489 the neurotransmitter profile of the neurons interspersed in the lumbar ventral white matter of

490 hop mice using in situ hybridization. GABA/glycinergic, glutamatergic and cholinergic

491 neurons were identified by detecting mRNA encoding the vesicular inhibitory amino acid

492 transporter (VIAAT), the vesicular glutamate transport (Vglut2), or the vesicular

493 acetylcholine transporter (VAChT). We found both VIAAT and Vglut2 positive neurons in

494 the ventral funiculus of hop mice demonstrating that misplaced neurons can be either

495 excitatory or inhibitory (Fig. 3A,B,E,F,I,J). In addition, we found that central canal

496 cholinergic cells were ventrally and medially displaced in hop mice compared to controls

497 (Fig. 3C,G), whereas no apparent differences were found regarding position and number of 498 motor neurons (Fig. 3D,H,K).

499 Ventromedial CINs with axons crossing in the ventral commissure are necessary and

500 sufficient for left-right coordination (Kjaerulff and Kiehn, 1996), which prompted us to

501 investigate the CINs in hop mice. Short- and long-range projecting CINs were examined by

502 tracer applications of fluorescein dextran amine (FDA) and rhodamine dextran amine (RDA)

503 in early postnatal mice (Fig. 4A). Midline retrograde tracing, using FDA to identify locally

504 CINs, revealed that CINs at the lumbar level 2 (L2, Fig. 4C,I) cluster together on the ventral

505 midline of hop mice compared to the two separate clusters of CINs seen in control mice (Fig.

$5064 \mathrm{~B}, \mathrm{H}$ ), whereas similar numbers of local CINs were found (Fig. 4N). FDA application on one

507 side of the L2 spinal cord illustrate a similar clustering of short projecting CINs on the ventral

508 midline as well as aberrant fiber crossing (4D,E). Despite altered positioning (Fig. 4J,K), the 22 
number of short-range projecting CINs in hop were similar compared to controls (Fig. 4O).

To study the long-range projecting CINs, we examined three different subpopulations; the intersegmental ascending (RDA), descending (FDA), and bifurcating (RDA/FDA) CINs (Fig 4F-P). In the cervical spinal cord (Fig. 4-1), and in control mice also at the lumbar level, our tracings experiments showed that long-range projecting CIN populations clustered in the ventromedial area. At the lumbar level in hop mice, however, CINs were diffusely dispersed and partly located on the midline (Fig. 4L,M). In addition, lumbar long-range projection CINs were found to be reduced in hop compared to control mice (Fig. 4P).

Taken together, these results show that, in hop mice, the ventromedial inhibitory and excitatory CINs responsible for normal left-right coordination cluster together on the ventral midline, and that intersegmental CINs are partly lost and misplaced.

\section{An early developmental defect underlies the altered functionality in hop mice}

CPG activity in rodents develops between embryonic day (E)12 and birth. Correct assembly and maturation results in an established left-right coordination circuitry around birth (Branchereau et al., 2000; Nakayama et al., 2002; Nishimaru and Kudo, 2000). The prominent ventral spinal cord phenotype observed in postnatal hop mice prompted us to investigate the onset of the morphological phenotype. Immunohistochemistry on E15.5 hop spinal cord tissue using microtubule associated protein 2 (Map2), to visualize fibers, and NeuN, to visualize neurons, revealed a similar fused spinal cord phenotype as seen in adult mice (Fig. 5A-D). In addition, using RDA tracing at E15.5 and E12.5, we found that the traceable CIN population in hop embryos was misplaced towards the midline and at E15.5 was more dispersed throughout the ventromedial spinal cord (Fig. 5E-H). These data indicate deviant ventral spinal cord formation presumaby due to abberant developmental processeses, such as midline axon guidance. 

mutations involving midline axon guidance molecules, including Netrin1 and ephrinB3. The absence of the midline chemo-attractant Netrin1 leads to a complete switch from alternating to synchronous fictive locomotor activity (Rabe et al., 2009). Further, mutations in either the ligand ephrinB3 or the receptor EphA4 cause abnormal midline crossing of corticospinal axons and interneuron axons in the CPG, resulting in mice with a characteristic hopping gait phenotype (Kullander et al., 2003). To assess the expression of axon guidance molecules in the midline of hop mice, we performed in-situ hybridization experiments on E15.5 and E12.5 spinal cord tissue with Netrin1 and EphrinB3 mRNA probes. The results showed that Netrin1 expression levels were severely reduced in the lumbar ventral spinal cord of hop mice, with a clear loss in the floorplate itself whereas remaining expression could be observed in the part of the spinal cord, which could at least partly explain the physiological phenotype in hop mice. However, guidance of commissural axons is not entirely dependent of floor-platederived Netrin1(Moreno-Bravo et al., 2019), so the loss of Netrin1 in hop mice may relate more to earlier morphogenic events.

Next, we explored the expression of Shh, a key regulator of early development. Immunohistochemistry on E12.5 tissue with a Shh antibody revealed that the distance between the notochord and ventral spinal cord border was clearly decreased (Fig. 6A,B,G), and that Shh levels were reduced in both the notochord and spinal cord of hop mice (Fig. 6H). 
abnormal displaying disorganised cells and low Shh expression (Fig. 6A',B'). Furthermore, Shh levels were also reduced in hop mutant embryos at E10.5 (Fig. 6C,D).

A point mutation in the Ttc26 gene was found in a previous study of hop mice (Swiderski et al., 2014). Ttc26 has been classified as an intraflagellar transport (IFT) complex B protein (Follit et al., 2009). Studies conducted on other IFT mutants have revealed phenotypic defects similar to those in the hop mutant including ventral spinal cord patterning defects, loss of ventral cell types and polydactyly (Haycraft et al., 2007; Huangfu et al., 2003; Liu et al., 2005). Therefore, we evaluated protein expression of TTC26 using immunohistochemical analysis and antibodies against TTC26 in the early developing spinal cord (Fig. 6E,F). Despite the apparent improper shape and positioning of the notochord at E12.5 we found, that the truncated TTC26 protein was still detectable in hop mice (Fig. 6F,I). Loss of Shh in mice results in degeneration of the notochord, absence of a morphologically distinct floorplate and abnormal dorsoventral patterning (Chiang et al., 1996). Consequently, we analyzed embryonic spinal cords of hop mice using antibodies against transcription factors as markers for specific developmental subpopulations to assess patterning defects (Fig. 6J-V, Fig. 6-1 (Gross et al., 2002; Moran-Rivard et al., 2001)). Most striking, this analysis revealed that the ventral most V3 progenitor domain labeled by Nkx2.2, although present in controls was undetectable in hop mice (Fig. $6 \mathrm{~J}, \mathrm{~K}$ ). The complete loss of Nkx2.2 positive cells in hop mice was restricted to the lumbar level and was found to be less apparent with increasing distance in both rostral and caudal directions and again, these defects were not found in the thoracic region (Fig. 6V). Further, we used Brn3a to examine the dorsal subpopulations dI1- dI3 and dI5 interneurons, which were largely unaffected, except in the most ventral regions (Fig. 6L,M; S4A,F). Similarly, the dI4 - dI6 subpopulations labelled by Lbx1 (Fig. 6N,O; S4B,G) and the dI4, dI6, p0 and p4 subpopulations labelled by Pax2 (Fig. 6P,Q; S4C,H) were present and appeared normal, except for the ventral-most labelled cells, 25 
which were found in close vicinity to the midline in hop mice. Markers for ventral V0v cells labelled by Evx1 and for dI3 and motor neurons labelled with Isl1/2 identified cells residing on and close to the ventral midline in hop mice (Fig. 6S,U; S4I,J), which was not seen in controls (Fig. 6R,T; S4D,E). Thus, our results show that there is an early patterning defect specific to the lumbar spinal cord of hop mice, likely originating from a defect in the notochord early organizer.

\section{Validation of the hop mutation}

In the hop mouse, defects that are associated with early developmental patterning and Shh signalling are apparent, supporting the hypothesis that the identified point mutation in Ttc26 is the causative mutation, and in line with a previous study (Swiderski et al., 2014). Our whole exome sequencing of a hop mouse and of Ttc26 exon 15 in several heterozygous and homozygous individuals confirmed a C to A transversion at position 38,412,065 bp causing a premature stop codon and a truncated form of the TTC26 protein (Fig. 7-1). However, TTC26 truncated protein remains expressed and it is possible that other mutations are present in neighboring genes, which may cause the observed phenotype. In the hop mutation area and in the close vicinity to the Ttc26 gene, genes that have been associated with gait defects can be found. For example, Hipk2 has been associated to TGF-beta signaling, another influential spinal cord morphant that plays an essential role in the regulation of survival in the nervous system (Doxakis et al., 2004; Isono et al., 2006; Zhang et al., 2007). Ablation of Hipk2 generated mice with an ataxic-like phenotype implying that the Hipk2 gene may contribute to the hop phenotype (Anzilotti et al., 2015).

We sought to exclude such additional possible causative mutations and determine whether the underlying genetic cause in the hop mouse is solely due to the identified mutation in Ttc26. Using clustered regularly interspaced short palindromic repeat/CRISPR-associated 9 26 
(CRISPR/Cas9) technology, we introduced a point mutation within Ttc26 exon 15 at position 38,412,065 bp in wild type mice (Fig 7A). The generated CRISPR Ttc26 ${ }^{Y 430 X}$ mutant mice displayed polydactyly of all feet (Fig. 7B,C), highly reminiscent of hop mice. However, whereas previous reports found a partial lethality between E10.5 and birth for hop mutant mice, we failed to receive live born homozygous Ttc26 $6^{Y 430 X}$ pups (Bryan et al., 1977). Among 67 delivered pups from 9 heterozygous breedings, we received one live-born homozygous pup that died early. We therefore performed our phenotypic analysis of embryonic stages. CRISPR Ttc26 $6^{\text {Y430X }}$ mutant embryos showed a spinal cord phenotype similar to the hop mice (Fig. 7B-H). Ventral spinal cord morphology matched the hop mice, with a poorly delineated and dorsally shifted central canal (Fig. 7E,F). Furthermore, like in hop mutants, defects were regionalized to the lumbar spinal cord with the cervical region unaffected (Fig. 7D). NeuN immunolabeling to identify neurons corroborated that the border between the white and grey matter was poorly defined, as was found in hop mutants (Fig. 7G, H). There were also less myelinated structures and misplaced neurons within the white matter, resulting in an absent ventral funiculus (Fig. 7G). These findings demonstrate that the spinal cord phenotype found in hop mice was mirrored in Ttc26 ${ }^{Y 430 X}$ mice, at least for embryonic stages. Thus, the C-to-A point mutation in the Ttc26-gene, exon 15 is likely the sole cause for the observed spinal cord defects in hop mice.

Finally, we sought to investigate how two populations of importance for locomotion were affected: motor neurons and a portion of the dI6 inhibitory interneurons defined by Dmrt3 expression (Andersson et al., 2012; Perry et al., 2019). Immunohistochemistry using primary antibodies against vesicular acetylcholine transporter (VAChT) were used to label motor neurons in the lumbar part of Ttc26 ${ }^{\mathrm{Y} 430 \mathrm{X}}$ and control spinal cords at E16.5.

Quantification gave a mean value of $19.6 \pm 9.4$ motor neurons per hemi-section (51 hemisections counted) in the Ttc $26^{Y 430 X}$ spinal cord and $20.6 \pm 9.3$ motor neurons per hemi27 
634 section (57 hemisections counted) in the control, which was not significantly different

635 (independent-samples Student's t-test; $\mathrm{p}=0.60$ ). Examination of the motor neuron distribution

636 in the lumbar region of the Ttc26 $6^{\mathrm{Y} 430 \mathrm{X}}$ spinal cord revealed two distinct populations on each

637 side of the midline, similar to controls (Fig 7I, J). This indicated that, despite the midline

638 fusion in the ventral spinal cord, the left and right populations of motor neurons remained

639 separate, as was also found in the adult hop mutants (Fig. 3). In contrast, central canal

640 cholinergic cells, that are normally separated by the midline, was distributed closer to and

641 even on the midline in the Ttc26 $6^{\mathrm{Y} 430 \mathrm{X}}$ spinal cord (Fig 7I',J'). We next explored the

642 distribution of dI6 interneurons using anti-Dmrt3 antibodies. As expected, in controls they

643 were located medioventrally in the spinal cord and formed two distinct populations on each

644 side of the midline. However, in Ttc26 ${ }^{\text {Y430X }}$ spinal cords, the Dmrt3 positive cells were

645 clustered on or close to the midline and positioned further ventrally (Fig. 7K,L,K',L').

646 


\section{Discussion}

648 Here we analyzed hop mice, focusing on the underlying cause for their aberrant locomotor 649 phenotype. Behavioral tests and fictive locomotion experiments showed that hop mice move 650 using their hindlimbs in synchrony. Our developmental studies revealed an abnormal 651 notochord, a missing floorplate and the absence of a Nkx2.2 progenitor domain in hop mice 652 as well as a disorganized dorsoventral patterning with neurons residing on the ventral midline. 653 Further, we found reduced expression of the axon guidance molecules netrin-1 and ephrinB3. 654 By verifying the causative mutation in Ttc26, we revealed its important role for the formation 655 of a lumbar locomotor network containing two separate CPG half-centers.

657 Lack of ventral spinal cord midline explains the synchronous gait

658 According to the half-centre model of the spinal locomotor CPG proposed by Graham-Brown 659 (Graham-Brown T, 1911), the rhythmic pattern of alternating bursts of flexor and extensor 660 muscles is produced by neuronal populations in each half of the spinal cord. Commissural 661 projections by inhibitory and excitatory neurons ensure normal left-right coordination (Kiehn, 662 2006). After cutting the ventral commissure the normal left-right circuit disappears indicating 663 that ventromedially located CINs are indispensable for the bilateral coordination (Kjaerulff 664 and Kiehn, 1996; Restrepo et al., 2009). Our morphological studies of the spinal cord in hop 665 mice revealed defects in the ventral spinal cord, including an absent midline and a loosely 666 defined white/grey matter border. The disturbed expression of axon guidance molecules in 667 hop mice are likely to underlie the observed aberrant fibres of short-, long- and locally668 projecting INs. Furthermore, their cell bodies were spread on the ventral midline compared to 669 the two separate ventromedial clusters of commissural INs seen in control mice (Fig. 4). Also, 
both excitatory and inhibitory neurons were found to reside on the ventral midline (Fig. 3).

671 Midline defects were discernable as early as E12.5, where we found that ventral neuronal

672 progenitors in the lumbar spinal cord were located where the midline should have been (Fig.

673 6). These results indicate that in hop mice, the neurons that normally form two separate CPG

674 half-centres, instead intermingle. This would cause a functionally fused CPG center,

675 explaining the observed synchronous gait.

Expression of pre-pattern genes along the dorsoventral axis of the spinal cord is controlled by long range signals from the opposite poles of the neural tube. Sonic Hedgehog, Gli3 and Smoothened are among the most important molecules for ventral cell differentiation, with the most ventral progenitor domain requiring the highest concentration of Shh to develop normally (Briscoe and Ericson, 1999; Placzek, 1995). Already at E10.5, the most ventral Nkx2.2 progenitor domain, which gives rise to V3 neurons, was absent in the hop mice. V3 neurons are one of the major classes of excitatory commissural interneurons in the mouse spinal cord and are components of the locomotor network, with a role in establishing a stable and balanced locomotor rhythm (Danner et al., 2019; Zhang et al., 2008).

Motor neuron populations, however, were not fused at the midline in hop mice. Previous studies have shown that the upper edge of the Islet1 expressing motor column is dorsally shifted in Netrin-1 mutant spinal cords at E10 (Kim et al., 2015). In hop mice, at E12.5, cells expressing Islet1 were dispersed dorsally and clustered together on the ventral midline (Fig. 6-1). Likewise, Robo mutant embryos (E9.5-E10.5) have a pack of misplaced Islet $1^{+}$motor neurons within the floorplate. However, the ectopic Islet $1^{+}$cells of Robo mutants are no longer visible in the floorplate by E12.5. Our study of the Ttc26 ${ }^{Y 430 X}$ spinal cords at E16.5 suggest that decreased levels of Netrin-1 in fact do affect the initial positioning of motor neurons, but that compensatory mechanisms then correct the mis-placing before birth. We did not find a significant difference between the number of motor neurons in 30 
695 Ttc26 $6^{Y 430 X}$ and control spinal cords, which indicates that the misplaced neurons most likely

696 migrate back to their correct location, rather than undergo apoptosis. In addition to

697 Semaphorin and Slit signalling governed by the transcription factors Islet1 and Islet2 (Lee et

698 al., 2015; Mauti et al., 2007), motor neuron positioning is regulated by Reelin and Cadherin

699 signalling causing a secondary reorganization of motor columns into sub-clusters consisting

700 of motor pools (Demireva et al., 2011; Palmesino et al., 2010; Price et al., 2002). The

701 migration and positioning of motor neurons seems to be more strictly regulated than the

702 positioning of CINs, possibly explaining why motor neurons are not clustered on the ventral

703 midline of hop and Ttc26 $6^{\text {Y430X }}$ spinal cords. Further studies are needed to understand the exact

704 underlying mechanisms for the observed differences between motor neuron and interneuron

705 development in the ventral spinal cord.

\section{Rostrocaudal specificity of the midline fusion}

In addition to the previously reported characteristic hopping gait in hop mice (Johnson and

Hunt, 1971; Wojnowski et al., 1998), our gait analysis showed that adult hop mice alternated their forelimbs while they moved their hind limbs in synchrony. Further, we found that the pattern of flexor-extensor alternation during fictive locomotion was maintained following hemisection, suggesting that the circuitry coordinating ipsilateral alternation is intact in hop mutants (Fig. 1-1). However, we did observe a tendency for asymmetry in flexor and extensor alternation (Fig. 1H,1-1A), suggesting that a bias toward extension exists, possibly due to a reduction in inhibitory drive from the flexor-related module of the CPG (Kiehn et al., 2008). This may be due to a reduced number of inhibitory descending CINs, or alternatively,

717 ipsilateral flexor inhibitory projections onto extensor motoneurons. Even if we found a

718 reduced number of traceable intersegmental CINs, both these scenarios remain possible and

719 are not mutually exclusive. These findings suggest that the defect behind the characteristic 31 
hopping phenotype is limited to the lumbar spinal cord controlling the left-right alternation of

721 the hind limbs, and possibly descending INs that affect coordination. This is in accordance with the lumbar specific defects observed in Ttc26 mutant mice early in development.

What might be the possible explanation for the rostro-caudal specificity of the defect? Primary cilia and Shh signalling are critical for proper patterning of the neural tube along the dorsoventral axis (Dessaud et al., 2008; Tarchini et al., 2006), and TTC26 is required for accumulation of Gli at the ciliary tip (Swiderski et al., 2014). In Shh KO mice, motor neurons are not generated, however, in Shh and Gli3 double KO mice there is a rescue effect where motor neurons are generated predominantly in the lumbar region (Litingtung and Chiang, 2000). This substantiates the involvement of the Shh signaling pathway in differential development along the rostral-caudal axis, possibly due to an unknown caudal factor such as retinoic acid (Litingtung and Chiang, 2000). Interestingly, missense mutations in the ttc26 gene are more frequent in the rabbit compared to other mammals with alternating gaits (Fig. 7-2A). To investigate if rabbits might have a rearranged lumbar region in their spinal cord explaining their synchronous hind limb gait, we analyzed rabbit tissue. There were, however, no signs of ventral fusion in the postnatal lumbar spinal cord (Fig. 7-2B) and spinal neuron populations were clearly separated (Fig. 7-2C-G), suggesting that mutations in Ttc26 are not underlying the synchronous gait in rabbits.

\section{The mutation in Ttc26 is causative for the spinal cord fusion}

Shh is crucial for induction of both floorplate and dorsoventral patterning of the spinal cord (Chiang et al., 1996; Patten and Placzek*, 2000; Placzek, 1995), and in particular, defective secretion of Shh can result in the spinal cord developing without floorplate cells and V3 interneurons (Iulianella et al., 2018). Shh mutant mice have several defects in midline structures such as the notochord and cyclopia (Boije et al., 2012). A similar spinal cord 32 
745 phenotype is present in HNF-3 $\beta$ null mutant mice. HNF-3 $\beta$ is a transcription factor expressed

746 in the three midline organizing centers; the node, notochord and floorplate (Ang and Rossant, 747 1994).

748 Similar to Swiderski et al., 2014, our positional cloning analysis localized the hop mutation to 749 Ttc26. TTC26, a component of Intraflagellar Transport (IFT) complex B, is a structure 750 necessary for cilia formation and protein transport, and is important for Shh signaling. In 751 addition, Ttc26 (also named IFT56) regulates vertebrate developmental patterning by 752 maintaining IFTB complex integrity and ciliary microtubule architecture (Xin et al., 2017). 753 Moreover, cilia motility is important for embryonic left-right determination (Nonaka et al., 1998). Although it is likely that the found mutation is the causative one, we noticed some differences between our analysis and the Swiderski et al. (2014) study. The spinal cord ventralisation was found in both studies, but additionally, we found midline fusion defects as well as lack of the ventral midline and Nkx2.2 positive V3 progenitors in the lumbar spinal cord. These differences opened the possibility of other additional mutations in the hop mice. For example, $\mathrm{Gli1}^{-/-}$; $\mathrm{Gli2}^{+/-}$mice have a hopping gait (Park et al., 2000), and $\mathrm{Gli3}^{+/-}$mice have preaxial polydactyly (Hui and Joyner, 1993; Park et al., 2000). In addition, a similar distortion of notochord, midline fusion (E14) and missing Nkx2.2 domain has been observed in Gli1/2 double mutants. Also, in $\mathrm{Gli2}^{-/}$mouse embryos, the most ventral cells (the floor 763 plate) are not specified (Park et al., 2000).

The lower expression levels of Shh seen in hop mice could also be caused by mutations in upstream regulators of Shh, events that could take place earlier in development. As mentioned above, the HOX1 cluster, located on cytoband B3-C $>25.40 \mathrm{cM}$ in the vicinity of the hop region, could be affected in the hop mouse mutation. Mice with homozygous mutations in one or multiple members of the cluster display a range of phenotypes including

769 developmental defects in the skeletal, reproductive and nervous systems (Raines et al., 2013). 33 
770

771

772

773

774

775

776

777

778

779

780

781

782

783

784

785

Two other genes in the hop region are associated to gait defects or could explain a fused spinal cord midline. When the Braf gene is mutated it has been reported to cause developmental deficits such as craniofacial dysmorphism and extra digits (Moriya et al., 2015). Mice with a targeted mutation in the transcriptional cofactor homeodomain interacting protein kinase 2 (HIPK2) display abnormal gaits including shuffling, reduced width and short stride length (Zhang et al., 2007). For this reason, we introduced a point mutation in the Ttc26 gene, which produced a premature stop codon mimicking the hop mouse mutation.

Unfortunately, potentially due to different genetic backgrounds, unlike hop mutants, Ttc26 ${ }^{\mathrm{Y} 430 X}$ mutants displayed fully penetrant embryonic lethality why locomotor studies could not be performed. However, Ttc26 $6^{Y 430}$ mutant mice displayed the same spinal cord ventral midline fusion as was found in hop mice, suggesting that this point mutation can sufficiently explain the observed mutant phenotype.

\section{Conclusion}

Our findings suggest that the aberrant spinal cord phenotype observed in hop mice is the result of abnormal developmental processes, including induction from the notochord and Shh signalling. Despite the loss of the ventral midline, functional neuronal networks develop and form connections able to produce coordinated, albeit synchronous, activity. Our data support the two half-centre hypothesis by demonstrating that midline separation is crucial for leftright alternation of locomotion. To our knowledge, the hop mutant is the first model with a specific ventral spinal cord fusion, in which the components of the CPG can be studied and understood. 


\section{Acknowledgements}

795 We thank C-J. Rubin, L. Strömstedt, A. Reis and S.P. Yee for technical assistance. We are

796 also grateful to S. Weatherbee for discussions of earlier versions of this work, and to

797 Stockholms Vilt- och Skadedjursgrupp and S. Boucher from Labovet conseil for donated

798 rabbit tissue. Supported by grants from Vetenskapsrådet (2018-02750, KK; 2015-03283,

799 2020-03365, HB), Swedish Brain Foundation (FO2020-0228, KK; FO2020-0129, HB), Knut

800 and Alice Wallenberg foundation (KAW 2016.0361, LA), Canadian Institutes of Health

801 Research (PJT-148682, PW).

802 
804 Ackerman, S.L., Kozak, L.P., Przyborski, S.A., Rund, L.A., Boyer, B.B., and Knowles, 805 B.B. (1997). The mouse rostral cerebellar malformation gene encodes an UNC-5-like 806 protein. Nature 386, 838-842.

807 Akay, T., McVea, D.A., Tachibana, A., and Pearson, K.G. (2006). Coordination of fore 808 and hind leg stepping in cats on a transversely-split treadmill. Exp Brain Res 175, 211809222.

\section{Andersson, L.S., Larhammar, M., Memic, F., Wootz, H., Schwochow, D., Rubin, C.-J.,} Patra, K., Arnason, T., Wellbring, L., Hjälm, G., et al. (2012). Mutations in DMRT3 affect locomotion in horses and spinal circuit function in mice. Nature 488, 642-646.

Ang, S.L., and Rossant, J. (1994). HNF-3 beta is essential for node and notochord formation in mouse development. Cell 78, 561-574.

Anzilotti, S., Tornincasa, M., Gerlini, R., Conte, A., Brancaccio, P., Cuomo, O., Bianco, G., Fusco, A., Annunziato, L., Pignataro, G., et al. (2015). Genetic ablation of homeodomain-interacting protein kinase 2 selectively induces apoptosis of cerebellar Purkinje cells during adulthood and generates an ataxic-like phenotype. Cell Death Dis 6, e2004.

Baddeley, A., and Turner, R. (2005). spatstat : An R Package for Analyzing Spatial Point Patterns. J. Stat. Soft. 12.

Bannatyne, B.A., Edgley, S.A., Hammar, I., Jankowska, E., and Maxwell, D.J. (2003). Networks of inhibitory and excitatory commissural interneurons mediating crossed reticulospinal actions. Eur. J. Neurosci. 18, 2273-2284.

Boije, H., Harun-Or-Rashid, M., Lee, Y.-J., Imsland, F., Bruneau, N., Vieaud, A., Gourichon, D., Tixier-Boichard, M., Bed'hom, B., Andersson, L., et al. (2012). Sonic Hedgehog-signalling patterns the developing chicken comb as revealed by exploration of the pea-comb mutation. PLoS ONE 7, e50890.

Bracci, E., Ballerini, L., and Nistri, A. (1996). Localization of rhythmogenic networks responsible for spontaneous bursts induced by strychnine and bicuculline in the rat isolated spinal cord. J. Neurosci. 16, 7063-7076.

Branchereau, P., Morin, D., Bonnot, A., Ballion, B., Chapron, J., and Viala, D. (2000). Development of lumbar rhythmic networks: from embryonic to neonate locomotor-like patterns in the mouse. Brain Res. Bull. 53, 711-718.

Briscoe, J., and Ericson, J. (1999). The specification of neuronal identity by graded Sonic Hedgehog signalling. Semin. Cell Dev. Biol. 10, 353-362.

Bryan, J.H.D., Hughes, R.L., and Bates, T.J. (1977). Brain development in hydrocephalic-polydactyl, a recessive pleiotropic mutant in the mouse. Virchows Arch. A Path. Anat. and Histol. 374, 205-214. 
840 Butt, S.J.B., and Kiehn, O. (2003). Functional identification of interneurons responsible 841 for left-right coordination of hindlimbs in mammals. Neuron 38, 953-963.

842 Chiang, C., Litingtung, Y., Lee, E., Young, K.E., Corden, J.L., Westphal, H., and Beachy, 843 P.A. (1996). Cyclopia and defective axial patterning in mice lacking Sonic hedgehog 844 gene function. Nature 383, 407-413.

845 Choi, M., Scholl, U.I., Ji, W., Liu, T., Tikhonova, I.R., Zumbo, P., Nayir, A., Bakkaloğlu, 846 A., Ozen, S., Sanjad, S., et al. (2009). Genetic diagnosis by whole exome capture and 847 massively parallel DNA sequencing. Proc. Natl. Acad. Sci. U.S.A. 106, 19096-19101.

848 Cowley, K.C., and Schmidt, B.J. (1995). Effects of inhibitory amino acid antagonists on 849 reciprocal inhibitory interactions during rhythmic motor activity in the in vitro neonatal 850 rat spinal cord. J. Neurophysiol. 74, 1109-1117.

851 Crone, S.A., Quinlan, K.A., Zagoraiou, L., Droho, S., Restrepo, C.E., Lundfald, L., Endo, 852 T., Setlak, J., Jessell, T.M., Kiehn, O., et al. (2008). Genetic ablation of V2a ipsilateral 853 interneurons disrupts left-right locomotor coordination in mammalian spinal cord. 854 Neuron 60, 70-83.

855 Crone, S.A., Zhong, G., Harris-Warrick, R., and Sharma, K. (2009). In mice lacking V2a 856 interneurons, gait depends on speed of locomotion. J. Neurosci. 29, 7098-7109.

857 Danner, S.M., Zhang, H., Shevtsova, N.A., Borowska-Fielding, J., Deska-Gauthier, D., 858 Rybak, I.A., and Zhang, Y. (2019). Spinal V3 Interneurons and Left-Right Coordination 859 in Mammalian Locomotion. Front Cell Neurosci 13, 516.

860 Demireva, E.Y., Shapiro, L.S., Jessell, T.M., and Zampieri, N. (2011). Motor neuron 861 position and topographic order imposed by $\beta$ - and $\gamma$-catenin activities. Cell 147, 641862652.

863 Dessaud, E., McMahon, A.P., and Briscoe, J. (2008). Pattern formation in the vertebrate 864 neural tube: a sonic hedgehog morphogen-regulated transcriptional network.

865 Development 135, 2489-2503.

866 Doxakis, E., Huang, E.J., and Davies, A.M. (2004). Homeodomain-interacting protein 867 kinase-2 regulates apoptosis in developing sensory and sympathetic neurons. Curr. Biol. 868 14, 1761-1765.

869 Drew, T., and Doucet, S. (1991). Application of circular statistics to the study of 870 neuronal discharge during locomotion. J. Neurosci. Methods 38, 171-181.

871 Eide, A.L., and Glover, J.C. (1995). Development of the longitudinal projection patterns 872 of lumbar primary sensory afferents in the chicken embryo. J. Comp. Neurol. 353, 247873259.

874 Fazeli, A., Dickinson, S.L., Hermiston, M.L., Tighe, R.V., Steen, R.G., Small, C.G., 875 Stoeckli, E.T., Keino-Masu, K., Masu, M., Rayburn, H., et al. (1997). Phenotype of mice 876 lacking functional Deleted in colorectal cancer (Dcc) gene. Nature 386, 796-804. 
Follit, J.A., Xu, F., Keady, B.T., and Pazour, G.J. (2009). Characterization of mouse IFT complex B. Cell Motil. Cytoskeleton 66, 457-468.

Gordon, I.T., and Whelan, P.J. (2006). Deciphering the organization and modulation of spinal locomotor central pattern generators. J. Exp. Biol. 209, 2007-2014.

Graham-Brown $T$ (1911). The intrinsic factors in the act of progression in the mammal. Proc. R. Soc. Lond. B 84, 308-319.

Gross, M.K., Dottori, M., and Goulding, M. (2002). Lbx1 specifies somatosensory association interneurons in the dorsal spinal cord. Neuron 34, 535-549.

Hammar, I., Bannatyne, B.A., Maxwell, D.J., Edgley, S.A., and Jankowska, E. (2004).

The actions of monoamines and distribution of noradrenergic and serotoninergic contacts on different subpopulations of commissural interneurons in the cat spinal cord. Eur. J. Neurosci. 19, 1305-1316.

Haycraft, C.J., Zhang, Q., Song, B., Jackson, W.S., Detloff, P.J., Serra, R., and Yoder, B.K. (2007). Intraflagellar transport is essential for endochondral bone formation. Development 134, 307-316.

Hinckley, C., Seebach, B., and Ziskind-Conhaim, L. (2005). Distinct roles of glycinergic and GABAergic inhibition in coordinating locomotor-like rhythms in the neonatal mouse spinal cord. Neuroscience 131, 745-758.

Hollander, W. (1976). Hydrocephalic-polydactyl, a recessive pleiotropic mutant in the mouse and its location in chromosome 6. Subject Strain Bibliography 1976 13-23.

Huangfu, D., Liu, A., Rakeman, A.S., Murcia, N.S., Niswander, L., and Anderson, K.V. (2003). Hedgehog signalling in the mouse requires intraflagellar transport proteins. Nature 426, 83-87.

Hui, C.C., and Joyner, A.L. (1993). A mouse model of greig cephalopolysyndactyly syndrome: the extra-toesJ mutation contains an intragenic deletion of the Gli3 gene. Nat. Genet. 3, 241-246.

Inui, M., Miyado, M., Igarashi, M., Tamano, M., Kubo, A., Yamashita, S., Asahara, H., Fukami, M., and Takada, S. (2015). Rapid generation of mouse models with defined point mutations by the CRISPR/Cas9 system. Sci Rep 4, 5396.

Isono, K., Nemoto, K., Li, Y., Takada, Y., Suzuki, R., Katsuki, M., Nakagawara, A., and Koseki, H. (2006). Overlapping roles for homeodomain-interacting protein kinases hipk1 and hipk2 in the mediation of cell growth in response to morphogenetic and genotoxic signals. Mol. Cell. Biol. 26, 2758-2771.

Iulianella, A., Sakai, D., Kurosaka, H., and Trainor, P.A. (2018). Ventral neural patterning in the absence of a Shh activity gradient from the floorplate. Dev. Dyn. 247, 170-184. 
Jankowska, E. (2008). Spinal interneuronal networks in the cat: elementary components. Brain Res Rev 57, 46-55.

Johnson, D.R., and Hunt, D.M. (1971). Hop-sterile, a mutant gene affecting sperm tail development in the mouse. J Embryol Exp Morphol 25, 223-236.

Juvin, L., Simmers, J., and Morin, D. (2005). Propriospinal circuitry underlying interlimb coordination in mammalian quadrupedal locomotion. J. Neurosci. 25, 60256035.

Kiehn, O. (2006). Locomotor circuits in the mammalian spinal cord. Annu. Rev. Neurosci. 29, 279-306.

Kiehn, O., Quinlan, K.A., Restrepo, C.E., Lundfald, L., Borgius, L., Talpalar, A.E., and Endo, T. (2008). Excitatory components of the mammalian locomotor CPG. Brain Res Rev 57, 56-63.

Kim, M., Fontelonga, T., Roesener, A.P., Lee, H., Gurung, S., Mendonca, P.R.F., and Mastick, G.S. (2015). Motor neuron cell bodies are actively positioned by Slit/Robo repulsion and Netrin/DCC attraction. Dev. Biol. 399, 68-79.

Kjaerulff, O., and Kiehn, O. (1996). Distribution of networks generating and coordinating locomotor activity in the neonatal rat spinal cord in vitro: a lesion study. J. Neurosci. 16, 5777-5794.

Kong, Y. (2011). Btrim: a fast, lightweight adapter and quality trimming program for next-generation sequencing technologies. Genomics 98, 152-153.

Kullander, K., Croll, S.D., Zimmer, M., Pan, L., McClain, J., Hughes, V., Zabski, S., DeChiara, T.M., Klein, R., Yancopoulos, G.D., et al. (2001). Ephrin-B3 is the midline barrier that prevents corticospinal tract axons from recrossing, allowing for unilateral motor control. Genes Dev. 15, 877-888.

Kullander, K., Butt, S.J.B., Lebret, J.M., Lundfald, L., Restrepo, C.E., Rydström, A., Klein, R., and Kiehn, O. (2003). Role of EphA4 and EphrinB3 in local neuronal circuits that control walking. Science 299, 1889-1892.

Lanuza, G.M., Gosgnach, S., Pierani, A., Jessell, T.M., and Goulding, M. (2004). Genetic identification of spinal interneurons that coordinate left-right locomotor activity necessary for walking movements. Neuron 42, 375-386.

Lee, H., Kim, M., Kim, N., Macfarlan, T., Pfaff, S.L., Mastick, G.S., and Song, M.-R. (2015). Slit and Semaphorin signaling governed by Islet transcription factors positions motor neuron somata within the neural tube. Exp. Neurol. 269, 17-27.

Lemieux, M., Laflamme, O.D., Thiry, L., Boulanger-Piette, A., Frenette, J., and Bretzner, F. (2016). Motor hypertonia and lack of locomotor coordination in mutant mice lacking DSCAM. J. Neurophysiol. 115, 1355-1371. 
949 Li, H., and Durbin, R. (2009). Fast and accurate short read alignment with Burrows-

950 Wheeler transform. Bioinformatics 25, 1754-1760.

951 Li, H., Handsaker, B., Wysoker, A., Fennell, T., Ruan, J., Homer, N., Marth, G., 952 Abecasis, G., Durbin, R., and 1000 Genome Project Data Processing Subgroup (2009).

953 The Sequence Alignment/Map format and SAMtools. Bioinformatics 25, 2078-2079.

954 Litingtung, Y., and Chiang, C. (2000). Specification of ventral neuron types is mediated 955 by an antagonistic interaction between Shh and Gli3. Nat. Neurosci. 3, 979-985.

956 Liu, A., Wang, B., and Niswander, L.A. (2005). Mouse intraflagellar transport proteins 957 regulate both the activator and repressor functions of Gli transcription factors.

958 Development 132, 3103-3111.

959 Lundfald, L., Restrepo, C.E., Butt, S.J.B., Peng, C.-Y., Droho, S., Endo, T., Zeilhofer, 960 H.U., Sharma, K., and Kiehn, O. (2007). Phenotype of V2-derived interneurons and their 961 relationship to the axon guidance molecule EphA4 in the developing mouse spinal cord.

962 Eur. J. Neurosci. 26, 2989-3002.

963 Lyon, M. (1973). Further data locating hpy in Linakge Group XI. Mouse News Lett. 49.

964 Madriaga, M.A., McPhee, L.C., Chersa, T., Christie, K.J., and Whelan, P.J. (2004).

965 Modulation of locomotor activity by multiple 5-HT and dopaminergic receptor subtypes

966 in the neonatal mouse spinal cord. J. Neurophysiol. 92, 1566-1576.

967 Mauti, O., Domanitskaya, E., Andermatt, I., Sadhu, R., and Stoeckli, E.T. (2007).

968 Semaphorin6A acts as a gate keeper between the central and the peripheral nervous 969 system. Neural Dev 2, 28.

970 Moran-Rivard, L., Kagawa, T., Saueressig, H., Gross, M.K., Burrill, J., and Goulding, 971 M. (2001). Evx1 is a postmitotic determinant of $v 0$ interneuron identity in the spinal 972 cord. Neuron 29, 385-399.

973 Moreno-Bravo, J.A., Roig Puiggros, S., Mehlen, P., and Chédotal, A. (2019). Synergistic 974 Activity of Floor-Plate- and Ventricular-Zone-Derived Netrin-1 in Spinal Cord 975 Commissural Axon Guidance. Neuron 101, 625-634.e3.

976 Moriya, M., Inoue, S.-I., Miyagawa-Tomita, S., Nakashima, Y., Oba, D., Niihori, T., 977 Hashi, M., Ohnishi, H., Kure, S., Matsubara, Y., et al. (2015). Adult mice expressing a 978 Braf Q241R mutation on an ICR/CD-1 background exhibit a cardio-facio-cutaneous 979 syndrome phenotype. Hum. Mol. Genet. 24, 7349-7360.

980 Nakayama, K., Nishimaru, H., and Kudo, N. (2002). Basis of Changes in Left-Right 981 Coordination of Rhythmic Motor Activity during Development in the Rat Spinal Cord. J. 982 Neurosci. 22, 10388-10398.

983 Nishimaru, H., and Kudo, N. (2000). Formation of the central pattern generator for 984 locomotion in the rat and mouse. Brain Res. Bull. 53, 661-669. 
Nonaka, S., Tanaka, Y., Okada, Y., Takeda, S., Harada, A., Kanai, Y., Kido, M., and Hirokawa, $N$. (1998). Randomization of left-right asymmetry due to loss of nodal cilia generating leftward flow of extraembryonic fluid in mice lacking KIF3B motor protein. Cell 95, 829-837.

Norreel, J.-C., Pflieger, J.-F., Pearlstein, E., Simeoni-Alias, J., Clarac, F., and Vinay, L. (2003). Reversible Disorganization of the Locomotor Pattern after Neonatal Spinal Cord Transection in the Rat. J. Neurosci. 23, 1924-1932.

Páez, P., Bátiz, L.-F., Roales-Buján, R., Rodríguez-Pérez, L.-M., Rodríguez, S., Jiménez, A.J., Rodríguez, E.M., and Pérez-Fígares, J.M. (2007). Patterned neuropathologic events occurring in hyh congenital hydrocephalic mutant mice. J. Neuropathol. Exp. Neurol. 66, 1082-1092.

Palmesino, E., Rousso, D.L., Kao, T.-J., Klar, A., Laufer, E., Uemura, O., Okamoto, H., Novitch, B.G., and Kania, A. (2010). Foxp1 and Lhx1 Coordinate Motor Neuron Migration with Axon Trajectory Choice by Gating Reelin Signalling. PLoS Biol 8, e1000446.

Park, H.L., Bai, C., Platt, K.A., Matise, M.P., Beeghly, A., Hui, C.C., Nakashima, M., and Joyner, A.L. (2000). Mouse Gli1 mutants are viable but have defects in SHH signaling in combination with a Gli2 mutation. Development 127, 1593-1605.

Patten, I., and Placzek*, M. (2000). The role of Sonic hedgehog in neural tube patterning: CMLS, Cell. Mol. Life Sci. 57, 1695-1708.

Perry, S., Larhammar, M., Vieillard, J., Nagaraja, C., Hilscher, M.M., Tafreshiha, A., Rofo, F., Caixeta, F.V., and Kullander, K. (2019). Characterization of Dmrt3-Derived Neurons Suggest a Role within Locomotor Circuits. J. Neurosci. 39, 1771-1782.

Placzek, M. (1995). The role of the notochord and floor plate in inductive interactions. Curr. Opin. Genet. Dev. 5, 499-506.

Price, S.R., De Marco Garcia, N.V., Ranscht, B., and Jessell, T.M. (2002). Regulation of motor neuron pool sorting by differential expression of type II cadherins. Cell 109, 205216.

Pruitt, K.D., Tatusova, T., Klimke, W., and Maglott, D.R. (2009). NCBI Reference Sequences: current status, policy and new initiatives. Nucleic Acids Res. 37, D32-36.

Quinlan, K.A., and Kiehn, O. (2007). Segmental, synaptic actions of commissural interneurons in the mouse spinal cord. J. Neurosci. 27, 6521-6530.

R Core Team (2021). R: A language and environment for statistical computing.

Rabe, N., Gezelius, H., Vallstedt, A., Memic, F., and Kullander, K. (2009). Netrin-1Dependent Spinal Interneuron Subtypes Are Required for the Formation of Left-Right Alternating Locomotor Circuitry. Journal of Neuroscience 29, 15642-15649. 
1021

1022

1023

1024

1025

1026

1027

1028

1029

1030

1031

1032

1033

1034

1035

1036

1037

1038

1039

1040

1041

1042

1043

1044

1045

1046

1047

1048

1049

1050

1051

1052

1053

1054

1055

1056

Rabe Bernhardt, N., Memic, F., Gezelius, H., Thiebes, A.-L., Vallstedt, A., and Kullander, K. (2012). DCC mediated axon guidance of spinal interneurons is essential for normal locomotor central pattern generator function. Dev. Biol. 366, 279-289.

Raines, A.M., Adam, M., Magella, B., Meyer, S.E., Grimes, H.L., Dey, S.K., and Potter, S.S. (2013). Recombineering-based dissection of flanking and paralogous Hox gene functions in mouse reproductive tracts. Development 140, 2942-2952.

Restrepo, C.E., Lundfald, L., Szabó, G., Erdélyi, F., Zeilhofer, H.U., Glover, J.C., and Kiehn, O. (2009). Transmitter-phenotypes of commissural interneurons in the lumbar spinal cord of newborn mice. J. Comp. Neurol. 517, 177-192.

Satoh, D., Pudenz, C., and Arber, S. (2016). Context-Dependent Gait Choice Elicited by EphA4 Mutation in Lbx1 Spinal Interneurons. Neuron 89, 1046-1058.

Schaeren-Wiemers, N., and Gerfin-Moser, A. (1993). A single protocol to detect transcripts of various types and expression levels in neural tissue and cultured cells: in situ hybridization using digoxigenin-labelled cRNA probes. Histochemistry 100, 431440.

Serafini, T., Colamarino, S.A., Leonardo, E.D., Wang, H., Beddington, R., Skarnes, W.C., and Tessier-Lavigne, M. (1996). Netrin-1 is required for commissural axon guidance in the developing vertebrate nervous system. Cell 87, 1001-1014.

Swiderski, R.E., Nakano, Y., Mullins, R.F., Seo, S., and Bánfi, B. (2014). A mutation in the mouse ttc26 gene leads to impaired hedgehog signaling. PLoS Genet. 10, e1004689.

Talpalar, A.E., Bouvier, J., Borgius, L., Fortin, G., Pierani, A., and Kiehn, O. (2013). Dual-mode operation of neuronal networks involved in left-right alternation. Nature 500, 85-88.

Tarchini, B., Duboule, D., and Kmita, M. (2006). Regulatory constraints in the evolution of the tetrapod limb anterior-posterior polarity. Nature 443, 985-988.

Thiry, L., Lemieux, M., and Bretzner, F. (2018). Age- and speed-dependent modulation of gaits in DSCAM2J mutant mice. J. Neurophysiol. 119, 723-737.

Vallstedt, A., and Kullander, K. (2013). Dorsally derived spinal interneurons in locomotor circuits. Ann. N. Y. Acad. Sci. 1279, 32-42.

Wallén-Mackenzie, A., Gezelius, H., Thoby-Brisson, M., Nygård, A., Enjin, A., Fujiyama, F., Fortin, G., and Kullander, K. (2006). Vesicular glutamate transporter 2 is required for central respiratory rhythm generation but not for locomotor central pattern generation. J. Neurosci. 26, 12294-12307.

Wegmeyer, H., Egea, J., Rabe, N., Gezelius, H., Filosa, A., Enjin, A., Varoqueaux, F., Deininger, K., Schnütgen, F., Brose, N., et al. (2007). EphA4-dependent axon guidance is mediated by the RacGAP alpha2-chimaerin. Neuron 55, 756-767. 
1057 Whelan, P., Bonnot, A., and O'Donovan, M.J. (2000). Properties of rhythmic activity 1058 generated by the isolated spinal cord of the neonatal mouse. J. Neurophysiol. 84, 282110592833.

1060 Wojnowski, L., Berna, R., Park, C.M., Handel, M.A., Hollander, W.F., and Zimmer, A. 1061 (1998). Reduced activity of BRAF protein kinase in hop and hop(hpy) mouse mutants.

1062 Mamm. Genome 9, 905-906.

1063 Wu, C.-Y., Gagnon, D.A., Sardin, J.S., Barot, U., Telenson, A., Arratia, P.E., and Kalb, 1064 R.G. (2018). Enhancing GABAergic Transmission Improves Locomotion in a 1065 Caenorhabditis elegans Model of Spinal Muscular Atrophy. ENeuro 5.

1066 Xin, D., Christopher, K.J., Zeng, L., Kong, Y., and Weatherbee, S.D. (2017). IFT56 1067 regulates vertebrate developmental patterning by maintaining IFTB complex integrity 1068 and ciliary microtubule architecture. Development 144, 1544-1553.

1069 Zagoraiou, L., Akay, T., Martin, J.F., Brownstone, R.M., Jessell, T.M., and Miles, G.B. 1070 (2009). A cluster of cholinergic premotor interneurons modulates mouse locomotor 1071 activity. Neuron 64, 645-662.

1072 Zhang, J., Pho, V., Bonasera, S.J., Holtzman, J., Tang, A.T., Hellmuth, J., Tang, S., 1073 Janak, P.H., Tecott, L.H., and Huang, E.J. (2007). Essential function of HIPK2 in 1074 TGFbeta-dependent survival of midbrain dopamine neurons. Nat. Neurosci. 10, 77-86.

Zhang, Y., Narayan, S., Geiman, E., Lanuza, G.M., Velasquez, T., Shanks, B., Akay, T., 1076 Dyck, J., Pearson, K., Gosgnach, S., et al. (2008). V3 spinal neurons establish a robust 1077 and balanced locomotor rhythm during walking. Neuron 60, 84-96. 


\section{Figure legends}

1080 Figure 1: Abnormal gait in hind limbs of hop mice (A) Gait analysis painting forelimbs (red) and

1081 hind limbs (blue) show that control mice move their fore- and hind limbs in an alternating pattern

1082 while hop mice move their hind limbs in a synchronous manner. Horizontal arrow L is the distance between the left and right paw and R the distance covered by the same right paw. (B) Graph showing quantification of walking pattern with the ratio L/R and significant genotype differences for hindlimb ( $p=0.021, r=-0.81)$ but not forelimb $(p=1, r=0)$ coordination ( $\mathrm{n}=4$ mice per group over a distance of $30 \mathrm{~cm}$ ). (C-E) hop mice show a characteristic synchronous left-right air-stepping pattern in vivo. (C) Schematic drawing of the experimental setup for recording from neonatal mice. (E,G) Raw EMG recordings from the left and right tibialis muscles of hop (G) and control (E) mice following a tail pinch stimulus illustrating representative synchronous and alternating traces, respectively ( $\mathrm{n}=2$ mice per group). The circular plots in (E,G) represent the phase of onset of the left and right tibialis anterior bursts with respect to each other. Each dot represents an individual burst, while the arrow indicates the tendency for the bursts to be coupled to each other. A phase value close to zero indicates synchrony in the case of the hop while a value close to $180^{\circ}$ suggests an alternating pattern in the case of the control animals ( $<$ 0.01). (D) Schematic illustration of ventral root recording setup measuring fictive locomotion. $(\mathrm{F}, \mathrm{H})$ The hop deficit can be reproduced in vitro using isolated spinal cord preparations. Control mice exhibit an alternating (F) and hop mice a synchronous (H) left-right pattern (n = 20 mice per group). Circular plots $(\mathrm{F}, \mathrm{H})$ indicate phase values across experiments. Each point equals one 60 second section of data (5 per animal). The length of the arrow provides an index of the strength of the coupling between the rhythms and the direction equals phase. (I-K) Bath application of GABA and glycinergic reuptake blockers affect the synchronous rhythm's stability in the hop mutant mouse.

1101 Control rhythm in hop mice (I) evoked by bath application of NMDA, 5-HT and DA. 20 minutes after adding nipecotic acid (J), one L2 root would double burst occasionally and at random. After application of nipecotic acid and sarcosine, occasional alternating segmental activity could be 
observed (K), although the ipsilateral L2-L5 rhythm was disrupted. For burst pattern following hemisection please refer to Fig 1-1. *Significant effect of genotype $p<0.05$.

Figure 2: Altered spinal cord morphology in postnatal hop mice. (A) Photomicrographs (ventral view) and (B) graph of measured sizes of early postnatal (p0-p5) cervical and lumbar spinal cords. At cervical levels from control and hop mice, no difference is apparent ( $p=1, r=0$; $\mathrm{n}=4$ animals per group). At lumbar levels, the lumbar enlargement of the spinal cord is less prominent in hop mice (black bar) compared to control mice (white bar, $p=0.009, r=-0.83$; $\mathrm{n}=5$ animals per group). The visible ventral spinal artery in control wild type mice (arrow) is absent from the lumbar part of the hop spinal cord (position noted with star). (C) Photomicrographs of immunohistochemistry staining with NeuN in red on spinal cord open-book preparations demonstrated lumbar specific defects in hop mice. White boxes indicate areas of higher magnification displayed in $C^{\prime}$. (C') Arrows point to the border between white matter and NeuN positive cells, and stars denote areas where aberrant NeuN positive cells are found. (D) Fluorescence intensity analysis of NeuN signal within region of interest as indicated (dotted lines in $\mathrm{C}^{\prime}$ ) reflects aberrant NeuN positioning in hop mice $(p=0.009, r=-0.83 ; \mathrm{n}=8$ per group). (E-H) Immunohistochemistry staining with MBP in green and NeuN in red, coupled to density plots in $(\mathrm{G}, \mathrm{H})$, shows misplaced neurons in the ventral spinal cord and a dorsally shifted central canal in hop mice at P5 (F, H) compared to control (E, G). (I) Peroxidase staining on $60 \mu \mathrm{m}$ transverse sections of adult spinal cords showed abnormal morphology in the lumbar spinal cord of hop mice. The central canal is dislocated dorsally and is smaller in size (arrowhead), the ventral commissure is missing and the ventral white matter is disorganized or absent (black arrow). (J-L) Close up and quantification of central canal phenotype. (K) Dorsal shift $(p \leq 0.001, d=3.88)$ and $(\mathrm{L})$ smaller size ( $p \leq 0.001, d=6.10)$ of central canal in hop mice $(\mathrm{n}=12)$ compared to controls $(\mathrm{n}=10)$. $(\mathrm{M}, \mathrm{N})$ Immunohistochemistry staining with NeuN in red and MBP in green confirms misplaced neurons in the grey matter of ventral spinal cord in hop mice at adult age $(\mathrm{N})$ compared to controls (M). (O) Quantification of number NeuN positive cells/section ( $\mathrm{n}=10$ per genotype) in the lumbar 
white matter in the hop adult mice compared to control $(p \leq 0.001, d=7.21)$. For brain morphology refer to Fig. 2-1. The dotted line in (E) indicates the midline, and in $(\mathrm{M}, \mathrm{N})$ the border between white and grey matter. Scale bars $300 \mu \mathrm{m}$ (A), $150 \mu \mathrm{m}$ (I), $100 \mu \mathrm{m}$ (J) and $50 \mu \mathrm{m}$ (C-E, M, N). Significant effect of genotype $* * p<0.01 * * * p \leq 0.001$.

Figure 3: Neurotransmitter phenotype in the ventral spinal cord of postnatal hop mice. (A-H) In situ hybridization on adult $60 \mu \mathrm{m}$ floating sections using probes against Viaat (A,E), Vglut2 (B,F) and $\operatorname{VAChT}(\mathrm{C}, \mathrm{D}, \mathrm{G}, \mathrm{H})$. In control mice, neurons marked with either Viaat (A) or Vglut2 (B) respect the border between white and grey matter, whereas in hop mice, Viaat (E) or Vglut2 (F) mRNA expressing neurons intermingle with the white matter. Region of interest (ROI) as indicated with dotted black line. (I) Number of displaced Viaat+ cells $(p<0.001, d=-2.31)$ and (J) Vglut2+ $(p<$ 0.001, $d=-3.16$ ) neurons in ROI are significant higher in lumbar but not cervical spinal cord ( $p \geq$ 0.05). VAChT stained cholinergic cells are found around the central canal in control (C) but are ventrally displaced relative to the central canal in hop mice (G). (D,H,K) Motor neuron position (LMC-MMC) and number are similar (K) in hop mice (D) and controls (H). $\mathrm{n}=11-13$ sections (L1 to L3) from n= 3 mice per genotype; Black box in (A) indicates area of enlargements. Neurons in the white matter are marked with black arrows. Arrowheads indicate central canal. Scale bars $50 \mu \mathrm{m}$ (AG), $150 \mu \mathrm{m}(\mathrm{D}-\mathrm{H})$. Significant effect of genotype $* * * p \leq 0.001$.

\section{Figure 4: Aberrant commissural fibres and interneuron positioning in the ventral lumbar spinal} cord of early postnatal (P0-P1) hop mice. (A) Schematics of different strategies for tracer application to detect commissural interneurons. Midline tracing using FDA to show local crossing commissural interneurons (B-C), local tracing using FDA to label short projecting CINs (D-E') or intersegmental FDA and RDA tracing to show ascending (aCINs), descending (dCINs) and bifurcating commissural interneurons (adCINs) in (F-G'). (B, C) Transverse sections within the L2 level of spinal cords and density maps (H, I) show interneuron clustering on the midline in hop mice (C, I) as 46 
1156

1157

1158

1159

1160

1161

1162

1163

1164

1165

1166

1167

1168

1169

1170

1171

1172

1173

1174

1175

1176

1177

1178

1179

1180

1181

opposed to two ventromedial clusters in controls (B, H). N) Quantification of traced cells show similar number of cells found ( $p \geq 0.05 ; n=9-12$ sections from 3 mice per genotype). (D-E') Transverse sections within the L2 level traced on L2 (D, E) shows that the fibres freely cross the midline in hop (E') compared to control mice (D'). (J, K) Density maps illustrate diffuse and aberrant positioning of CINs on the midline in hop mice compared to controls. O) Quantification of traced cells show similar CIN numbers ( $p \geq 0.05 ; \mathrm{n}=6-9$ sections from 3 mice per genotype. (F-G') Aberrant fibres cross over the midline in spinal cords of hop mice (G, G') ventral from the central canal and the amount of longrange projecting CINs are reduced compared to controls. (L,M) Density plots of aCINs (red) and dCINs (green) in control (L) and hop (M) spinal cords. (P) Quantification of traced cells show a significant reduction of intersegmental projections over the midline (RDA: $p \leq 0.001, d=2.92$, FDA: $p \leq 0.001, d=2.96$; aCIN, dCIN and adCIN: $p \leq 0.001, d=4.46 ; \mathrm{n}=8-11$ sections from 4 mice per genotype). For intersegmental tracing on cervical spinal refer to Fig. 4-1. White box in (D, F) indicates the area of close-up (D', G'). The outline of the spinal cord is indicated by a dashed line (B, C), central canal and midline are indicated by a dotted line (F', G'). Scale bars (B-G) $100 \mu \mathrm{m}$ and (D'-G') 200 $\mu \mathrm{m}$. Significant effect of genotype $* * * p \leq 0.001$.

\section{Figure 5: The distinct spinal cord phenotype in hop mice is present at embryonic stages. (A-B)}

Photomicrographs of immunohistochemistry staining on E15.5 hop and control spinal cords with

NeuN (green) and Map2 (red) showing aberrant fibres and misplaced neurons disrespecting the ventral midline. (C-D') White box in (A, B) indicates region of interest (ROI) for statistical analysis of aberrant positioned NeuN+ cells and fiber crossing (C, NeuN+ cells: $p<0.001, d=-5.46$; D', Map2 intensity as ratio to control: $p<0.001, d=-2.81$; $\mathrm{n}=5$ per genotype). White line in $\mathrm{A}^{\prime}$ and $\mathrm{B}^{\prime}$ indicates the thickness of the ventral commissure, quantified length as ratio to controls in (D) ( $p<0.001, d=$ 5.71; $n=5$ per genotype). (E-H) RDA retrograde commisural interneuron (CIN) tracing on E15.5 (E-F) and E12.5 (G-H) spinal cord. Dotted line indicates midline and outline of the spinal cord. (E'-H') Density plots visualizing medial displacement of traced CIN in hop ( $\mathrm{n}=12$ sections from 3 embryos 
per genotype). (I-P) In-situ hybridization on E15.5 (I, J, M, N) and E12.5 (K, L, O, P) lumbar spinal cords using probes detecting Netrin1 and EphrinB3. Arrow heads point to regions of ventral Netrin1 and EphrinB3 mRNA expression. (Q, R) Staining intensity (background corrected ratio) is significantly reduced in hop compared to control spinal cords for both axon guidance molecules and time points (Netrin; E12.5 $p<0.001, d=5.86, \mathrm{n}=7$ per group, E15.5: $p<0.001, d=3.47, \mathrm{n}=5$ per group; EphrinB3; E12.5 $p<0.001, d=6.46, \mathrm{n}=7$ per group, E15.5: $p<0.001, d=5.19, \mathrm{n}=10$ per group). Scale bars $50 \mu \mathrm{m}$ (A-H), $100 \mu \mathrm{m}$ (I-P). Significant effect of genotype ${ }^{* * *} p \leq 0.001$.

\section{Figure 6 Abnormal Shh and lumbar spinal cord patterning in hop mice}

(A-D) Photomicrographs of Shh immunohistochemistry staining on transverse spinal cord sections at E12.5 (A, B) and E10.5 (C, D). Notochord and floorplate phenotype at E12.5 in low power (A, B) and close up from control (A', A") and hop (B', B") spinal cords. Dashed lines outline the spinal cord and notochord. The distance between the notochord and spinal cord is indicated by stars (A, B) and is reduced in hop spinal cords (G; $p<0.001, d=7.23, \mathrm{n}=15$ per group). (A', B') A malformed notochord together with reduced amounts of Shh ( $\mathrm{H} ; p=0.002, d=2.50, \mathrm{n}=5$ per group). Lack of floorplate cells and severely reduced Shh expression in the spinal cord (A" ,B") of hop mice compared to controls (H), ( $p=0.016, d=1.93, \mathrm{n}=5$ per group). (C, D) Similarly, we found reduced amounts of Shh at E10.5 in hop spinal cords compared to controls (H), ( $p<0.001, d=1.36, \mathrm{n}=15$ per group). (E, F) Ttc26 immunostaining on E12.5 spinal cord transverse sections. For clarity, blue nuclear counterstain with DAPI has been omitted. (I) The expression of Ttc26 is found in the notochord at similar intensity levels both in control and hop spinal cords. (J-V) Immunostainings on E12.5 spinal cord sections reveal a missing Nkx2.2 progenitor domain and a migration defect of neurons in the lumbar spinal cord of hop mice. (J, K) The expression of the ventral most V3 progenitor marker Nkx2.2 is absent in lumbar sections from hop mice (K). White box indicates area of higher magnification (J', K'). (V) Nkx2.2 phenotype at different rostrocaudal levels exemplified with photomicrographs and visualized using density plots. The midline is indicated by lines ( $\mathrm{n}=4$ sections per level). ( $\mathrm{L}-\mathrm{V})$ Density plots 48 
1208 show misplaced cells and lack of a ventral midline for additional progenitor markers as specified

1209 below each panel (n=3), for representative photomicrographs refer to Fig. 6-1). (W) Schematic

1210 summary of defects found in the hop mice in the lumbar part of the spinal cord including weak Shh

1211 expression, misplaced and malformed notochord, a dorsally shifted central canal, absence of floorplate

1212 and p3 domains as well as absence of a ventral midline. pdINs - progenitor domains for dorsal

1213 interneurons, FP - floor plate. The outline of the spinal cord and midline are indicated by dashed lines.

$1214 \mathrm{FP}=$ floorplate, NC=notochord, scale bars: $100 \mu \mathrm{m}(\mathrm{A}-\mathrm{B}, \mathrm{J}-\mathrm{K}, \mathrm{M}-\mathrm{V}), 25 \mu \mathrm{m}\left(\mathrm{A}^{\prime}, \mathrm{B}^{\prime}\right) 30 \mu \mathrm{m}\left(\mathrm{A}^{\prime \prime}, \mathrm{B}^{\prime \prime}, \mathrm{J}\right.$ '

$\left.1215 \mathrm{~K}^{\prime}\right)$ and $50 \mu \mathrm{m}(\mathrm{C}, \mathrm{D})$. Significant effect of genotype ${ }^{*} p<0.05^{* *} p<0.01 * * * p \leq 0.001$.

1216

1217 Figure 7: Introduction of a point mutation in Ttc26 and analysis Ttc26 ${ }^{\text {Y430X }}$ mutants. For initial

1218 validation of the hop mouse mutation refer to Fig. 7-1. (A) We then applied the CRISPR/Cas9 system

1219 to generate mice with a point mutation in exon 15 of the Ttc26 gene, generating a substitution of

1220 tyrosine to a premature stop codon. By simultaneous microinjection of guideRNA, hCas9 mRNA and

1221 a single-stranded donor oligonucleotide (ssDON) into mouse oocytes, mouse chromosome 6

1222 harbouring the Ttc26 gene was modified (left). The exon (black) and intron (red) organization is

1223 indicated together with Sanger sequencing traces of wild type (bottom) and the mutated allele (top)

1224 highlighting the premature stop mutation. The introduction of a PvuII site for screening purposes

1225 (blue) and the location of the guideRNA are indicated. (B,C) Images of E12.5 (B) and E16.5 (C)

1226 Ttc26 ${ }^{Y 430 X}$ mice showing preaxial polydactyly (arrowheads, star). (D-F) Photomicrographs of

1227 Hematoxylin and Eosin staining on E16.5 transverse spinal cord sections. (D) Lumbar spinal cord of

1228 control mice showing a well delineated central canal (upper black arrow), a present ventral funiculus

1229 with a border between white and grey matter (dashed line) and a present ventral spinal artery (lower

1230 arrow). (E) Lumbar spinal cord of Ttc26 $6^{Y 430 X}$ mice showing a poorly delineated dorsally shifted central

1231 canal (upper arrow), an absent ventral funiculus (upper star) and an absent ventral spinal artery (lower

1232 arrow). (F) Cervical spinal cord of $T t c 26^{Y 430 X}$ mice showing a well delineated central canal (upper

1233 arrow) and a present ventral funiculus with a clear midline border (lower arrow). (G-L) 
1234 Photomicrographs of immunohistochemistry experiments on E16.5 transverse lumbar spinal cord

1235 sections of control and Ttc26 ${ }^{Y 430 X}$ mice. (G) NeuN staining showing a poorly defined ventral midline

1236 in Ttc26 ${ }^{Y 430 X}$ mice, with misplaced neurons within the white matter of the ventral funiculus.

1237 Enlargements on the right show cells positioned at the location of the missing midline in mutant but

1238 not control spinal cord sections (G-H). (I-J) Cholinergic neurons as identified by their VAChT

1239 expression (red) were found in the medial and lateral motor neuron cluster, as well as in neurons

1240 around the central canal. Dmrt3 expressing interneurons were found medioventrally from the central

1241 canal. In mutant Ttc26 $6^{Y 430}$ spinal cords, medial and lateral motor neuron nuclei were located at their

1242 expected positions. In contrast, central canal cholinergic neurons and Dmrt3 expressing interneurons

1243 were found ventral of the central canal and positioned in close proximity or even on top of the

1244 presumed position of the midline. (I, J) VAChT immunostaining showing no apparent differences

1245 regarding position and size of motor neurons between Ttc26 ${ }^{\mathrm{Y4} 30}$ and control mice. (I',J') Central canal

1246 VAChT expressing cells, as well as (K-L) Dmrt3 expressing cells, were ventrally and medially

1247 displaced in Ttc26 ${ }^{Y 430}$ spinal cords compared to controls. (J',L') Enlargement of the ventromedial area

1248 of the spinal cord demonstrating misplaced cells in Ttc26 $6^{Y 430}$ spinal cords (J', arrows). Scale bars (D-

1249 H) $100 \mu \mathrm{m}$, (I-L) $50 \mu \mathrm{m}$, (I'-L') $100 \mu \mathrm{m}$. For comparative analysis in the rabbit refer to Fig. 7-2.

1250 
Figure 1-1 (related to figure 1): Mid-sagittal hemisection does not disrupt the ipsilateral alternating burst pattern. (A) Neurograms illustrating a typical pattern recorded from a hop mouse.

(B) Pattern recorded from the preparation following a midsagittal hemisection. Note the preservation of the L3-L5 pattern suggesting that flexor-extensor coordination is maintained. (C) Schematic illustrating stimulation and recording arrangement for cauda equina stimulation. Cauda equina evoked rhythmic pattern (4 Hz, $10 \mathrm{~s}$ train) showed segmental synchronous pattern and ipsilateral alternating

1259 pattern.

Figure 2-1 (related to figure 2): Brain commissure morphology appears normal in hop mice. tissue. (A,B) The corpus callosum (CC) and hippocampal commissure (HC) showed no gross defects. (C,D,G,H) The anterior commissure (AC) also appeared normal. (E,F) Sections showing the habenular commissure (HaC), the posterior commissure (PC) and the subcommissural organ (sc), all three present and with normal appearance in both control and hop mice. (I,J) The pontine nuclei (pn) is also present in both genotypes. $(\mathrm{K}, \mathrm{L})$ No aberrant commissure was found between the roof of the fourth ventricle (FV), and cerebellum (ce) at the junction of midbrain and hindbrain, in control and hop mice. Scale bars (A,B,C,D,G,H) $1000 \mu \mathrm{m},(\mathrm{E}, \mathrm{F}, \mathrm{I}, \mathrm{J}, \mathrm{K}, \mathrm{L}) 500 \mu \mathrm{m}$

Figure 4-1 (related to figure 4): Normal commissural interneurons and axon midline crossing in the cervical spinal cord of early postnatal (P0-P1) hop mice. (A-F) Intersegmental tracing using FDA/RDA to locate ascending (aCINs), descending (dCINs) and bifurcating commissural interneurons (adCINs). No apparent defects in hop mice (D-F') compared to controls (A-C') on cervical sections were found. 
Figure 6-1 (related to figure 6): Embryonic Patterning defects in hop spinal cords.

1278 Photomicrographs of immunohistochemistry experiments on E12.5 spinal cord transverse sections

1279 using antibodies against transcription factors as indicated at the top. White boxes in (A-E) indicate

1280 areas of higher magnification in the respective panel below (A'-E'). The stainings show misplaced

1281 cells and lack of ventral midline for all tested progenitor markers. The outline of the spinal cord,

1282 central canal and midline are indicated by dashed lines. Scale bars (A-J) $100 \mu \mathrm{m},\left(\mathrm{A}^{\prime}, \mathrm{F}^{\prime}\right) 50 \mu \mathrm{m}$ and

1283 (B', G'-J') $30 \mu \mathrm{m}$.

1284

1285 Figure 7-1 (related to figure 7): Validation of the hop mouse mutation. The exon/intron

1286 organization of Ttc26 is indicated together with Sanger sequencing traces of wild type and hop

1287 highlighting the premature stop mutation in exon 15 (out of 18) of Ttc26. Genomic DNA from a hop

1288 homozygote (stock 002718) and BALB/cByJ control (stock 001026) was obtained from the Jackson

1289 Laboratory's DNA Resource (n=3 per genotype). Exomes were captured at the Yale Center for

1290 Genome Analysis, using the NimbleGen SeqCap EZ Mouse Exome and following NimbleGen

1291 protocols. Captured pools were sequenced (75bp, paired-end) on an Illumina HiSeq 2000 using

1292 previously described methods (Choi et al., 2009). We obtained 91 million (BALB/cByJ) to 113

1293 million (hop) high-quality reads. Illumina reads were first trimmed based on their quality scores to

1294 remove low-quality regions using the program Btrim (Kong, 2011). A cutoff of 20 for average quality

1295 scores within a moving window of size 5-bp was used. Minimum acceptable read length was 25-bp.

1296 Other parameters of Btrim were set to defaults. The pre-processed reads were then aligned to the

1297 mouse genome reference sequence (mm9) using the BWA mapping program (Li and Durbin, 2009).

1298 The mapping results were converted into SAMtools pileup format using SAMtools programs (Li et al.,

1299 2009). PCR duplicates were removed using the rmdup command from SAMtools, resulting in $~ 84 \mathrm{x}$

1300 (BALB/cByJ) or 100x (hop) coverage across the exome. >97\% of all bases included in the exome

1301 showed at least $8 x$ coverage and $>90 \%$ of the bases showed at least $20 x$ coverage. Single nucleotide

1302 variations (SNVs) were called using SAMtools' pileup command. Further filtering was performed

1303 using in-house scripts to exclude those SNV calls that had less than 3 reads or a SNP score less than 52 
1304 20. Annotation was added based on the UCSC RefSeq gene model (http://genome.ucsc.edu/ (Pruitt et

1305 al., 2009)). Based on SNV homozygosity mapping, the interval was narrowed to 700 Kb on

1306 Chromosome 6, (37.9-38.6 Mb) which fell within the previously mapped hop genomic region. After

1307 filtering for known SNPs and repeat regions, we identified 17 possible point mutations, 16 of which

1308 were located in introns or 3' untranslated regions.

1309

Figure 7-2 (related to figure 7): Relatively high number of missense mutations in the rabbit

1311 Ttc26 gene. (A) Amino acid alignment of TTC26 sequences from eight mammals made using

1312 MUSCLE with missense mutations in rabbit marked (pink boxes). (B) Photomicrograph of HE stained

1313 lumbar spinal cord section from wild type adult rabbit (box shown magnified region). The morphology

1314 is similar to the wild type mouse spinal cord, with a well delineated central canal (upper arrow), a

1315 ventral funiculus with a clear border between white and grey matter and a ventral spinal artery (lower

1316 arrow). (C-G) Immunohistochemistry performed on postnatal rabbits towards NeuN (C), Chat (D),

1317 Lbx1 (E), Parvalbumin (F) and Pax2 (G) show a clear bilateral separation of the spinal neuronal

1318 populations. Scale bars (C-G) $200 \mu \mathrm{m}$. 
A

G
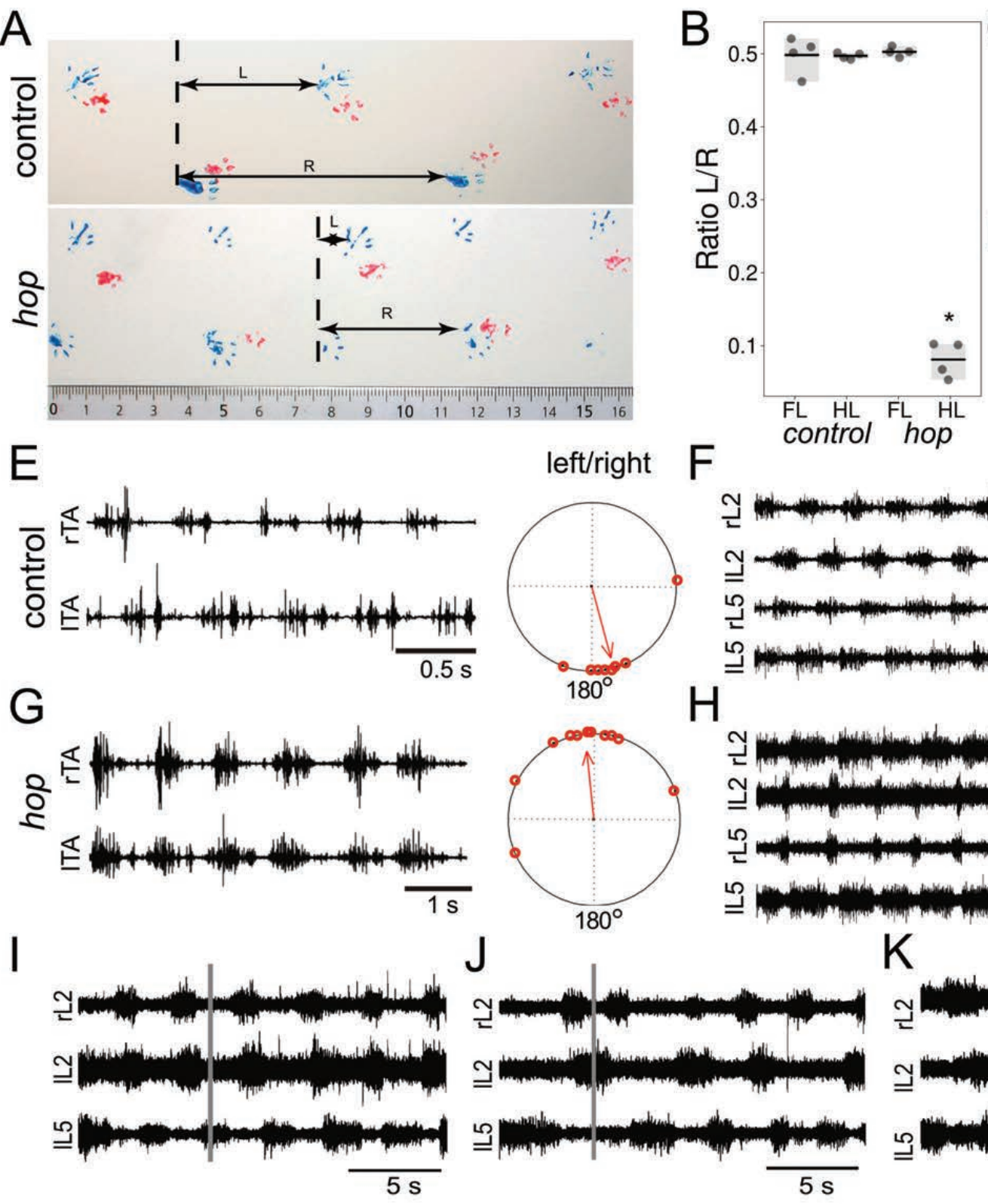

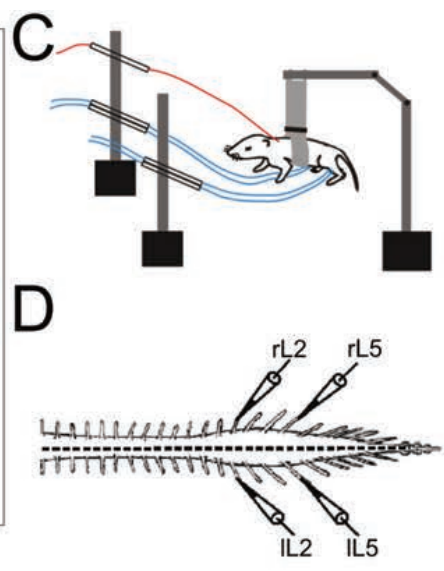

left/right
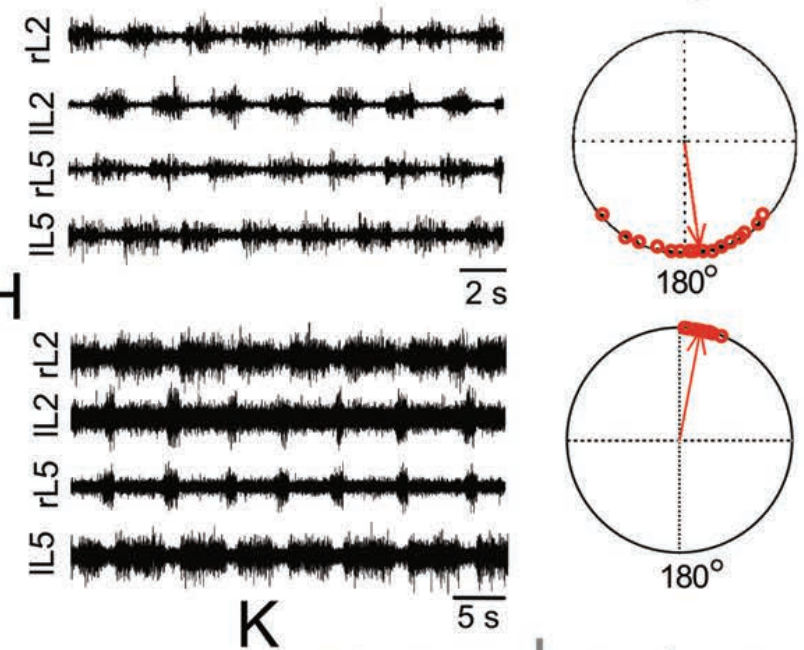

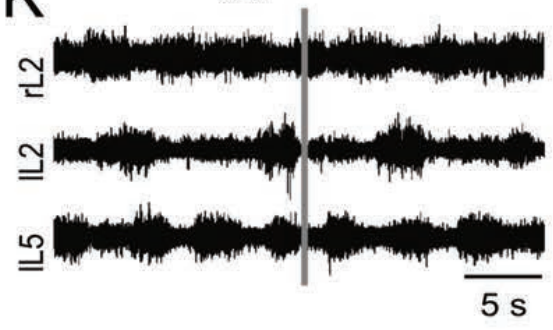


Figure 2
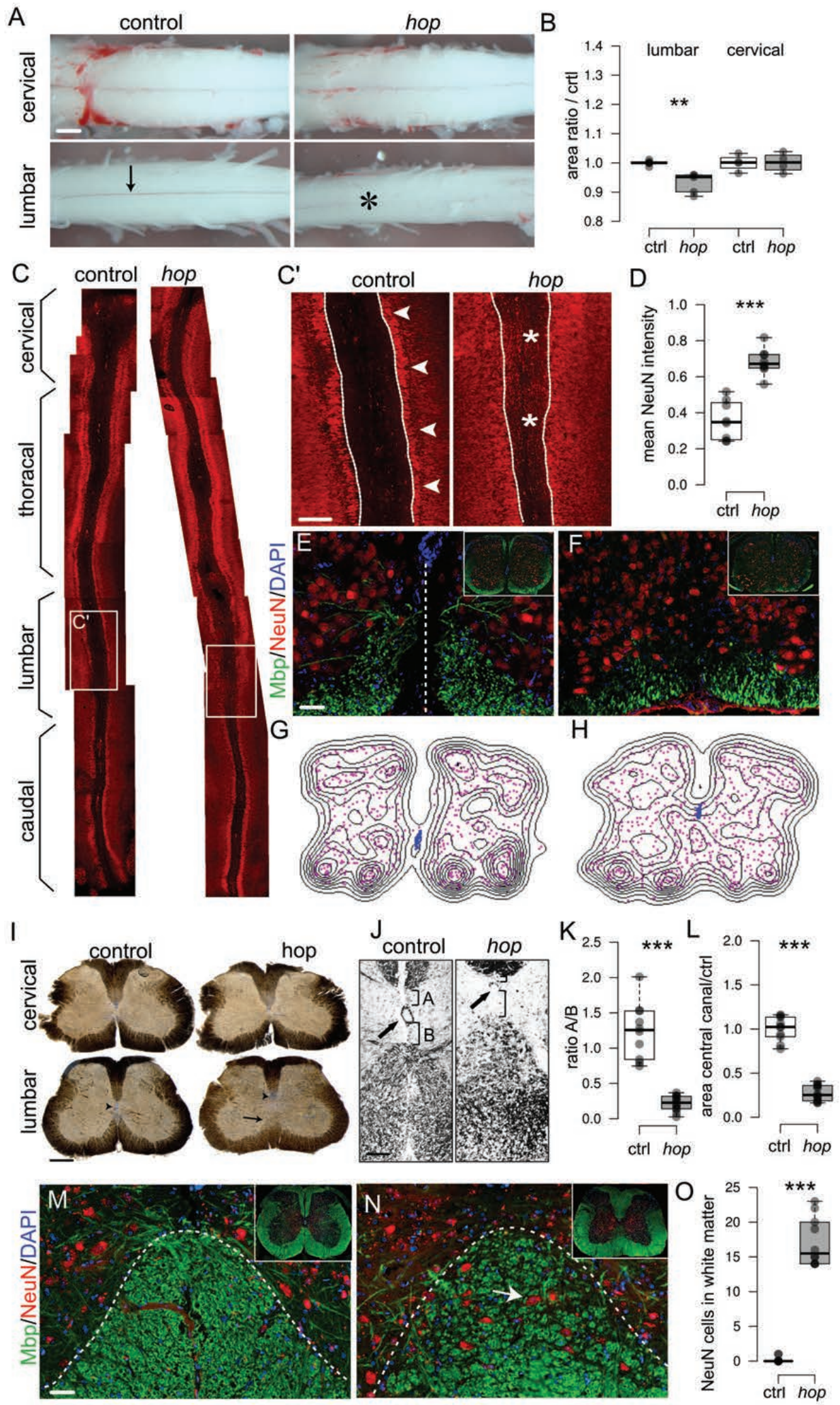
Figure 3
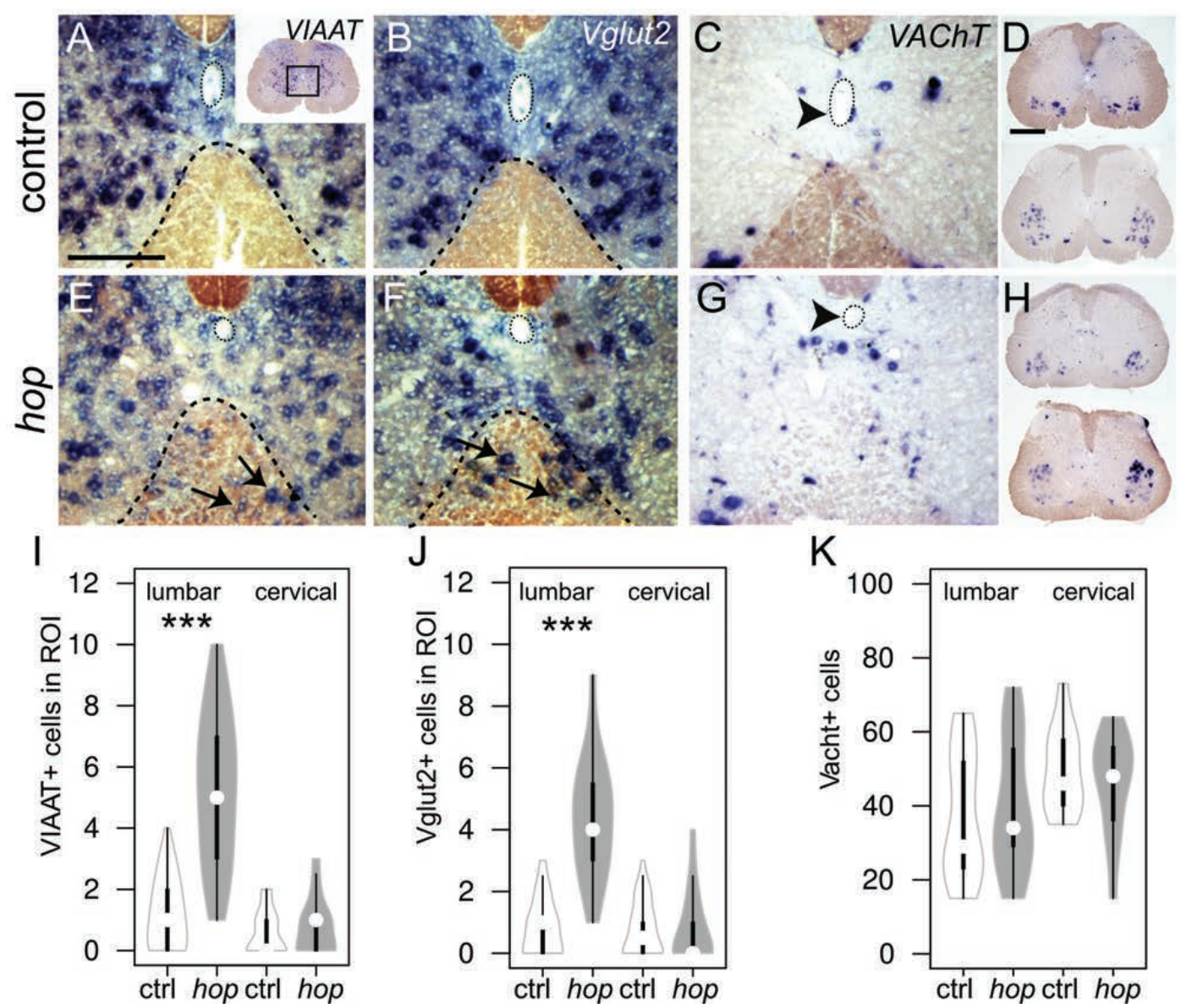
Figure 4
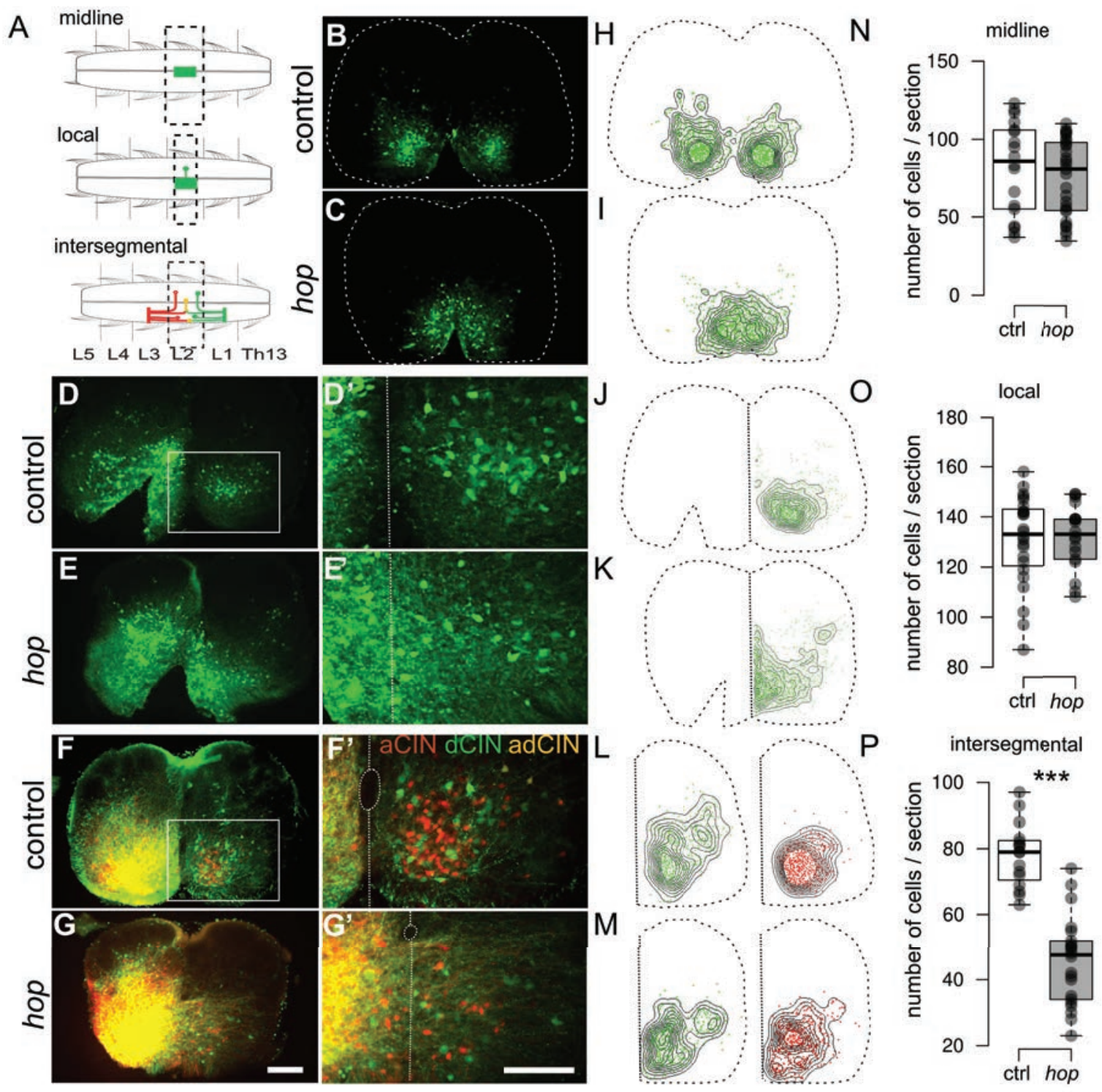


\section{Figure 5}

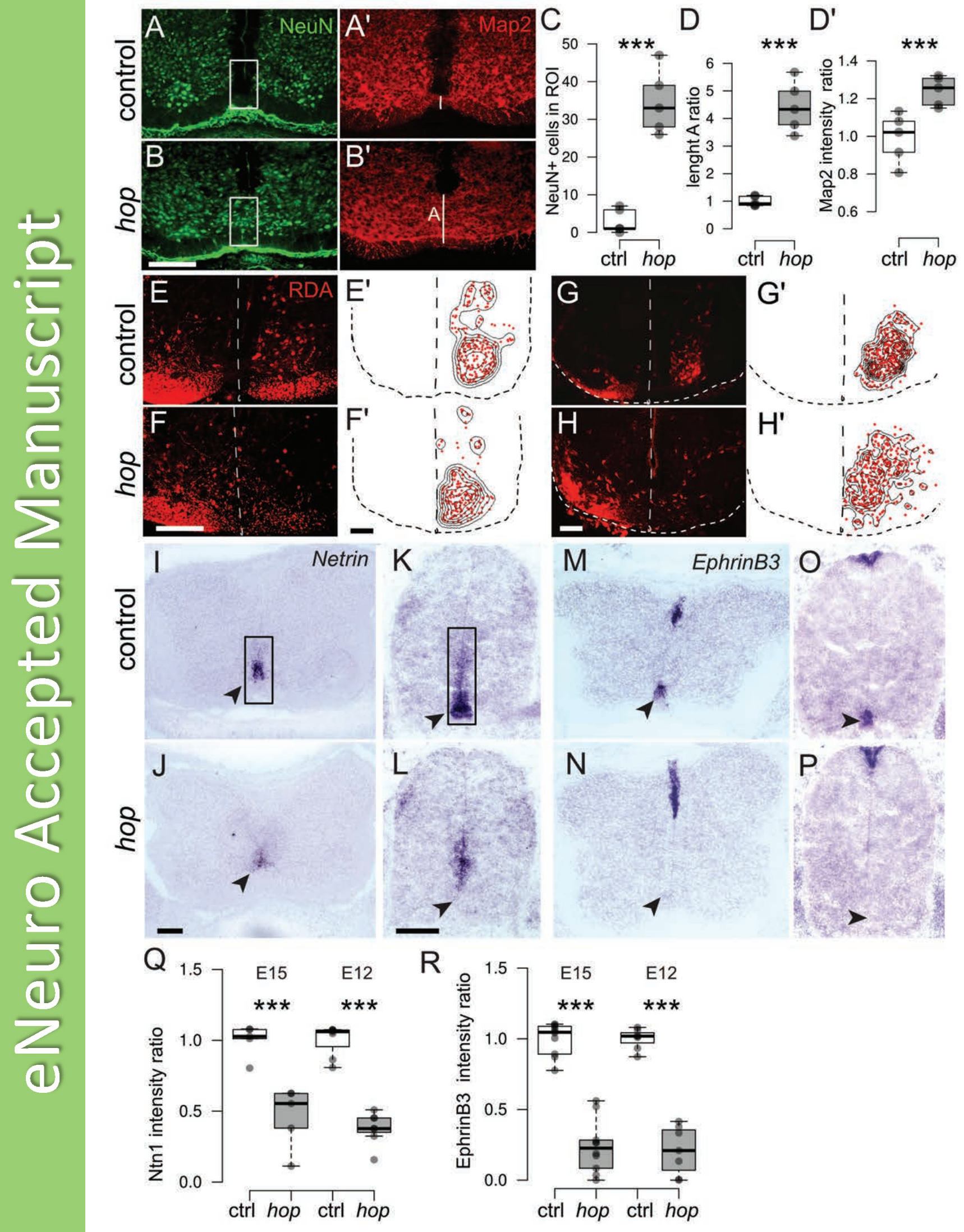


Figure 6
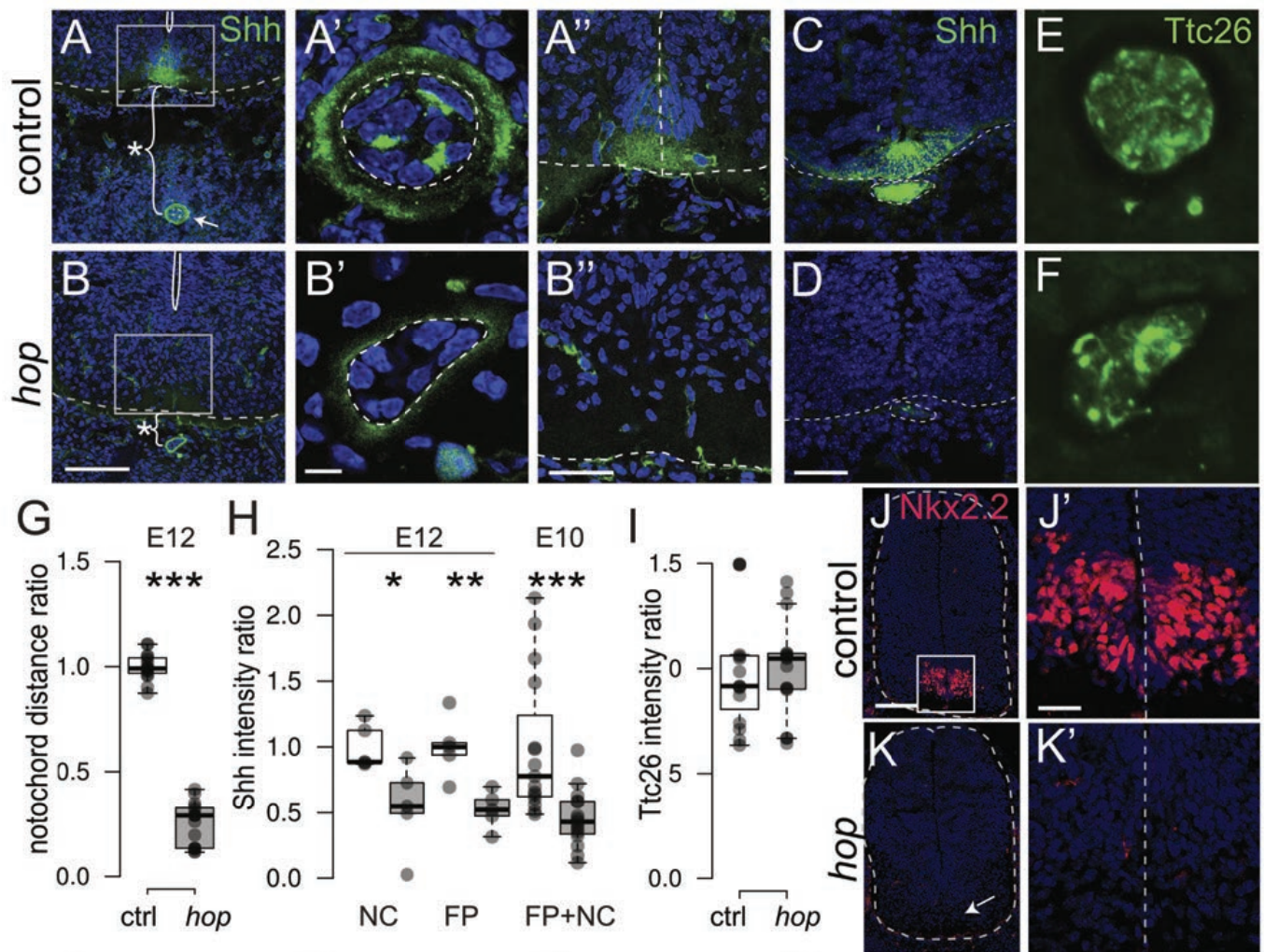

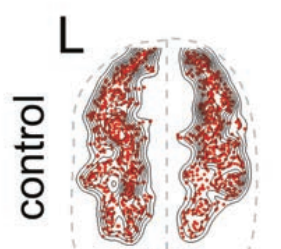

\section{$\mathrm{N}$}
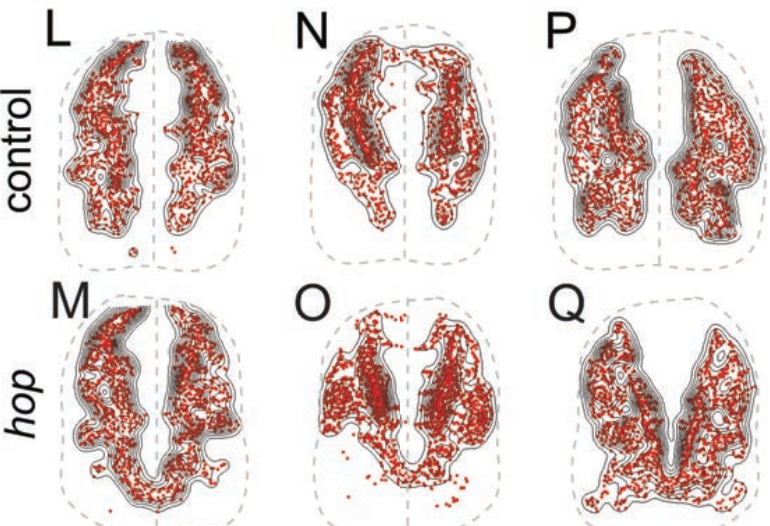

Brn3a
O

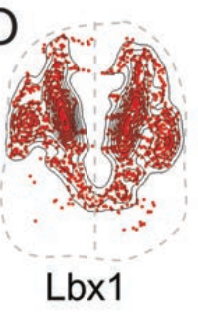

Q

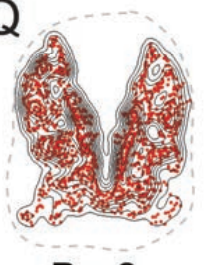

Pax2
$\mathrm{R}$
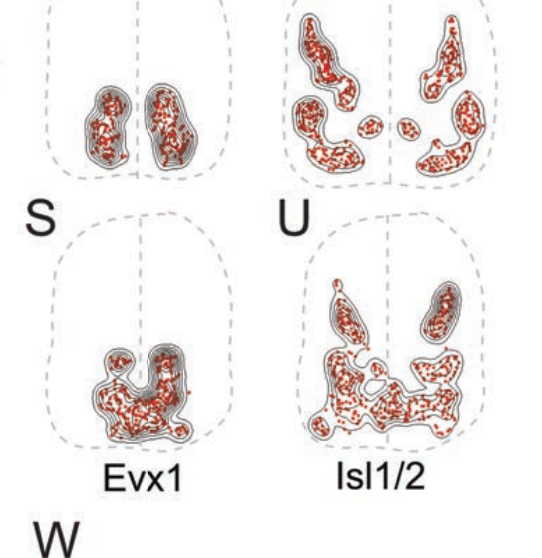
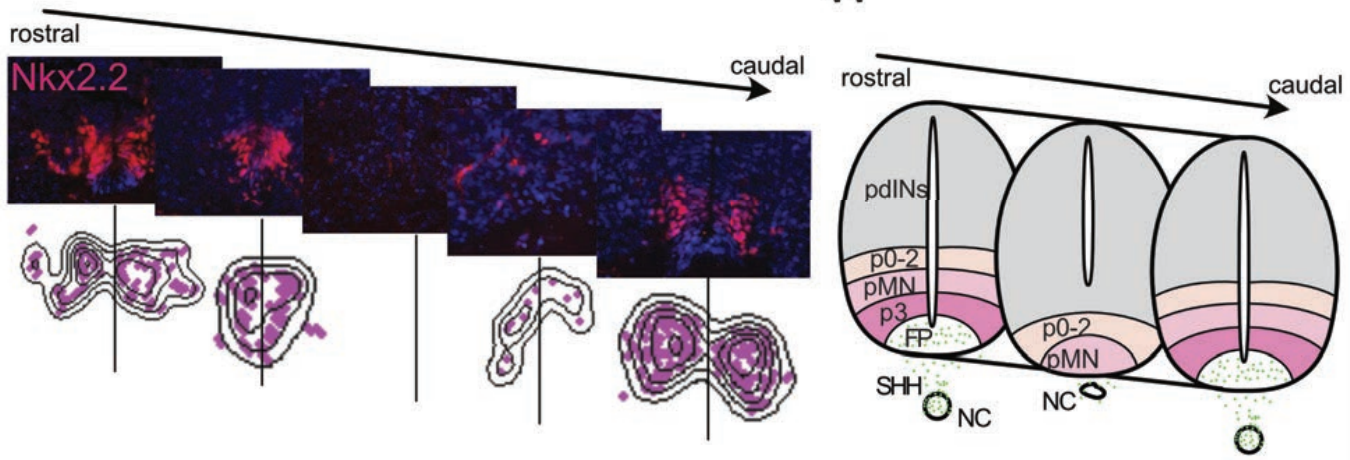


\section{Figure 7}

A
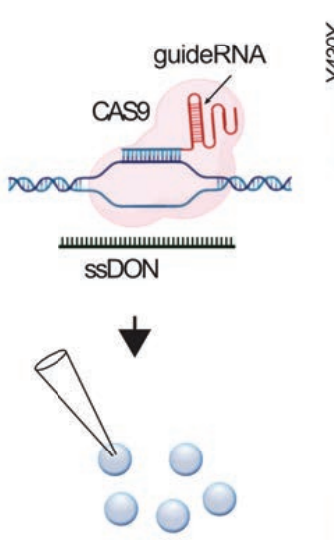

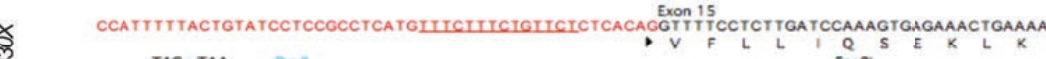

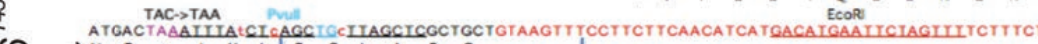

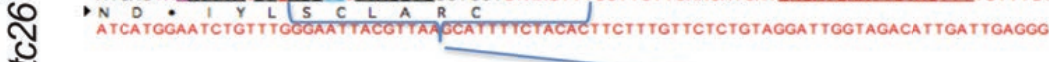
F

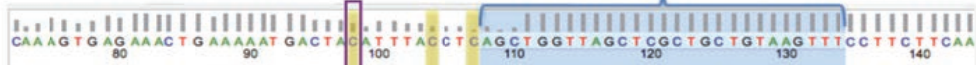

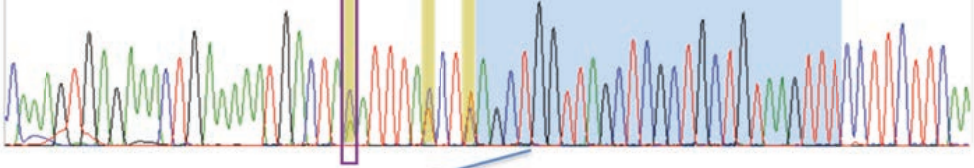

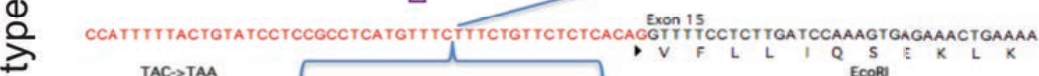

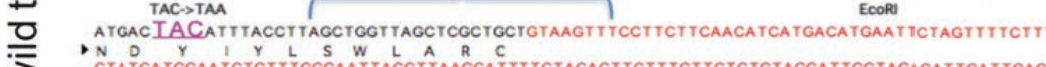

年
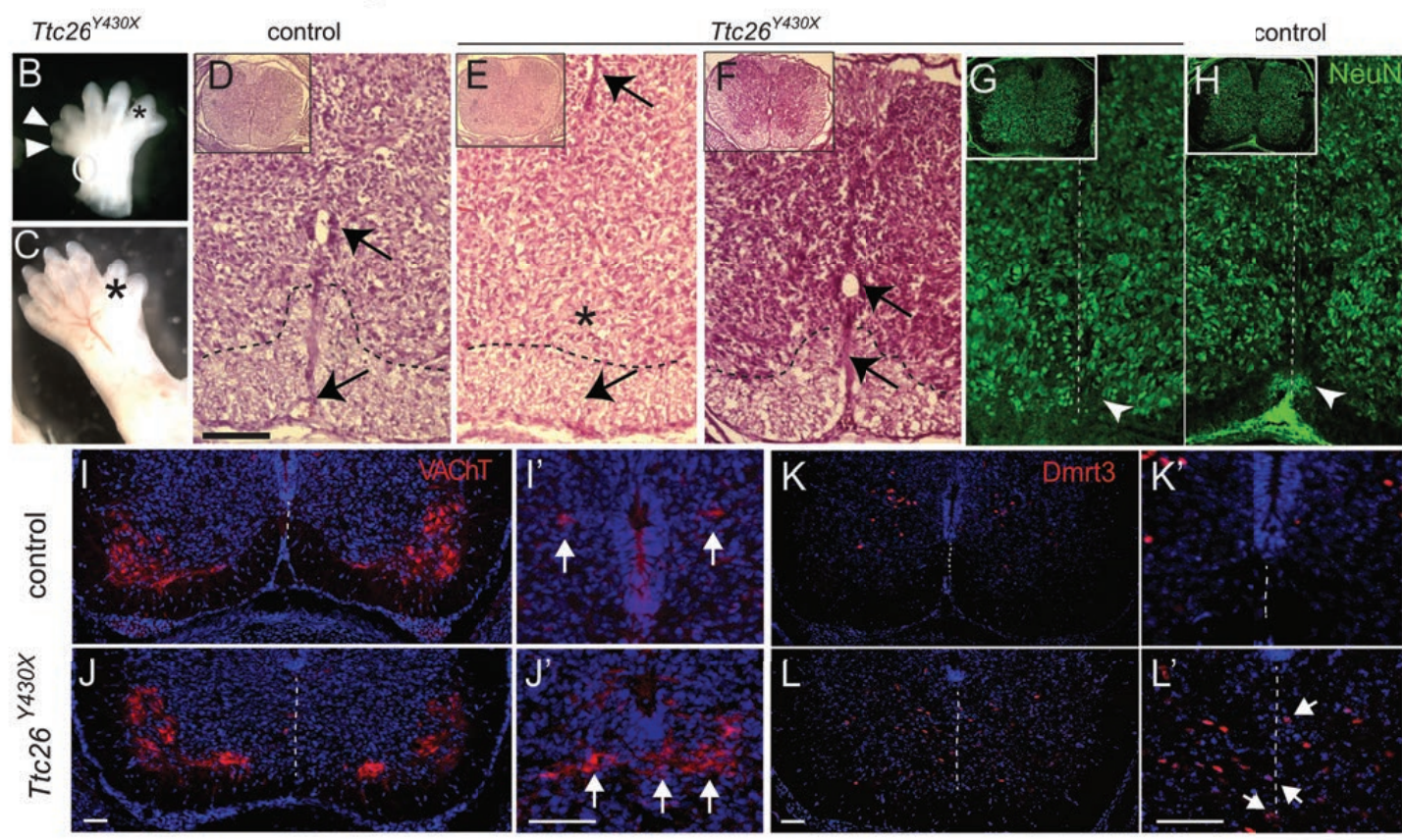